\title{
The logic of distributive bilattices
}

\author{
FÉLIX BOU, Institut d'Investigació en Intel.ligència Artificial, IIIA - \\ CSIC, Campus UAB, Bellaterra 08193, Spain.E-mail: fbou@iiia.csic.es \\ UMBERTO RIVIECCIO, Department of Philosophy, University of \\ Genoa, Italy.E-mail: umberto.rivieccio@unige.it
}

\begin{abstract}
Bilattices, introduced by Ginsberg [26] as a uniform framework for inference in Artificial Intelligence, are algebraic structures that proved useful in many fields. In recent years, Arieli and Avron [3] developed a logical system based on a class of bilattice-based matrices, called logical bilattices, and provided a Gentzen-style calculus for it. This logic is essentially an expansion of the well-known Belnap-Dunn four-valued logic to the standard language of bilattices. Our aim is to study Arieli and Avron's logic from the perspective of Abstract Algebraic Logic (AAL). We introduce a Hilbert-style axiomatization in order to investigate the properties of the algebraic models of this logic, proving that every formula can be reduced to an equivalent normal form and that our axiomatization is complete w.r.t. Arieli and Avron's semantics. In this way we are able to classify this logic according to the criteria of AAL. We show, for instance, that it is non-protoalgebraic and non-selfextensional. We also characterize its Tarski congruence and the class of algebraic reducts of its reduced generalized models, which in the general theory of AAL is usually taken to be the algebraic counterpart of a sentential logic. This class turns out to be the variety generated by the smallest non-trivial bilattice, which is strictly contained in the class of algebraic reducts of logical bilattices. On the other hand, we prove that the class of algebraic reducts of reduced models of our logic is strictly included in the class of algebraic reducts of its reduced generalized models. Another interesting result obtained is that, as happens with some implicationless fragments of well-known logics, we can associate with our logic a Gentzen calculus which is algebraizable in the sense of Rebagliato and Verdú [33] (even if the logic itself is not algebraizable). We also prove some purely algebraic results concerning bilattices, for instance that the variety of (unbounded) distributive bilattices is generated by the smallest nontrivial bilattice. This result is based on an improvement of a theorem by Avron [6] stating that every bounded interlaced bilattice is isomorphic to a certain product of two bounded lattices. We generalize it to the case of unbounded interlaced bilattices (of which distributive bilattices are a proper subclass).
\end{abstract}

Keywords: Bilattice, many-valued logic, abstract algebraic logic, reduced model, non-protoalgebraic logic, algebraizable Gentzen system. 


\section{The logic of distributive bilattices}

\section{Introduction and Preliminares}

Bilattices are algebraic structures introduced by Ginsberg [26] as a uniform framework for inference in Artificial Intelligence, in particular within default and non-monotonic reasoning. In the last two decades these structures have proved useful in many fields, of which we shall here mention just a few.

Bilattices were extensively investigated by Fitting, who considered applications to Logic Programming [15, 16] (on this topic see also [27, 28]), to philosophical problems such as the theory of truth $[14,19]$ and studied the relatioship with a family of manyvalued systems generalizing Kleene's three-valued logics [17, 18]. Other interesting applications include the analysis of entailment, implicature and presupposition in natural language [34], the semantics of natural language questions [31] and epistemic $\operatorname{logic}[35]$.

In the nineties bilattices were investigated in depth by Arieli and Avron, both from an algebraic $[5,6]$ and a logical point of view $[2,4]$. In order to deal with paraconsistency and non-monotonic reasoning in Artificial Intelligence, Arieli and Avron [3] developed the first logical system in the traditional sense based on bilattices. This logic, which which we shall call $\mathcal{L B}$, is defined semantically from a class of matrices called logical bilattices, and is essentially an expansion of the well known Belnap-Dunn four-valued logic to the standard language of bilattices. In [3] a Gentzen-style calculus is presented as a syntactic counterpart of $\mathcal{L B}$, and completeness and cut elimination are proved. Our main concern in the following sections will be to investigate $\mathcal{L B}$ from the point of view of Abstract Algebraic Logic (which we abbreviate AAL). For all definitions and general results from AAL that we will use, the reader is referred to $[13,22]$.

Besides the relationship with the aforementioned bilattice-based formal systems, one of our main interests in this logic comes from the fact that it is one of the few known natural examples of what in AAL are called non-protoalgebraic logics.

Recall that a logic $\langle\mathcal{L}, \vdash\rangle$ is said to be protoalgebraic (see $[9,13])$ if and only if there is a set $\Delta(p, q)$ of formulas (in two variables) such that $\vdash \Delta(p, p)$ and $\{p, \Delta(p, q)\} \vdash q$. Roughly speaking, the class of protoalgebraic logics is the broadest class of logical systems that are relatively well-behaved from an algebraic point of view. One of the great open issues in AAL is whether it is possible to obtain for non-protoalgebraic logics results concerning the connection between a logic and its associated class of algebras that may be compared to those that have been established for the protoalgebraic ones.

Some examples of non-protoalgebraic logics are the implicationless fragment of classical and intuitionistic logics, modal logics with strict implication and many-valued logics preserving lower bounds of degrees of truth. One of the reasons why a satisfying general theory is still missing is that relatively few examples of non-protoalgebraic logics have been studied up to now. We believe that the study of particular logics belonging to this class may help to better understand the non-protoalgebraic landscape and in the long run might allow to improve the general theory of their algebraization.

The paper is organized as follows. In the remaining part of this section we recall some basic definitions and facts concerning bilattices and the logic $\mathcal{L B}$ of Arieli and Avron (for proofs and details the reader is referred to $[15,16,3]$ ).

In Section 2, in order to investigate the properties of the algebraic models of $\mathcal{L B}$, we introduce a Hilbert-style calculus which is an extension of the one given by Font 


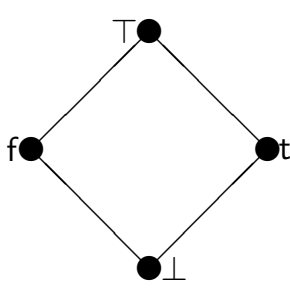

$\mathcal{F O U R}$

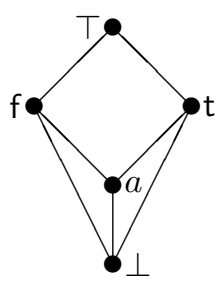

$\mathcal{F I V E}$

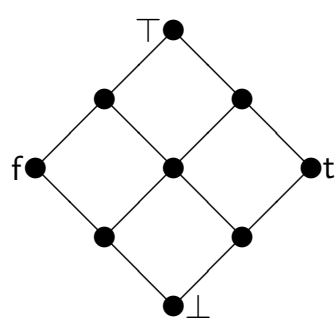

$\mathcal{N} \mathcal{I N E}$

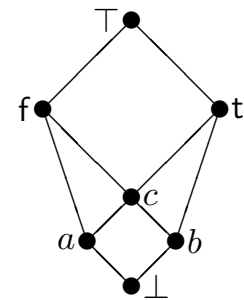

$\mathcal{D E F} \mathcal{A} \mathcal{U} \mathcal{L}$

FIG. 1. Some examples of (pre-)bilattices

[20] for the Belnap-Dunn logic. We establish a normal form theorem for our calculus and, using this result, prove completeness with respect to the semantics of $\mathcal{L B}$.

In Section 3 we prove some purely algebraic results concerning bilattices that will later be used to study $\mathcal{L B}$ from the perspective of AAL: among these is a generalization of a well-known representation theorem, stating that every interlaced bilattice is isomoprhic to a certain product of two lattices.

In Section 4 we investigate in depth the algebraic models of $\mathcal{L B}$. We characterize the classes of reduced models and of reduced generalized models of $\mathcal{L B}$, as well as the corresponding classes of algebraic reducts $\operatorname{Alg}{ }^{*} \mathcal{L B}$ and $\operatorname{Alg} \mathcal{L B}$. Finally, we consider a slight variant of the Gentzen-style calculus introduced by Arieli and Avron [3]: we show that this calculus is algebraizable in the sense of Rebagliato and Verdú [33] with respect to the variety of distributive bilattices, which is also the class of algebraic reducts of reduced generalized models of $\mathcal{L B}$.

The terminology concerning bilattices is not uniform ${ }^{1}$. In this section we explain the terminology used in the present paper. We adopt the convention of using the name "bilattice" when there is a negation operator, since this has become more or less standard in recent papers about bilattices.

A pre-bilattice is an algebra $\mathbf{B}=\langle B, \wedge, \vee, \otimes, \oplus\rangle$ such that $\langle B, \wedge, \vee\rangle$ and $\langle B, \otimes, \oplus\rangle$ are both lattices. The order associated with the lattice $\langle B, \wedge, \vee\rangle$ is denoted by $\leq_{t}$ and called the truth order, while the order $\leq_{k}$ associated with $\langle B, \otimes, \oplus\rangle$ is the knowledge order. Usually in the literature it is required that the lattices be complete or at least bounded, but here none of these assumptions is made. The minimum and maximum of the truth lattice, in case they exist, will be denoted by $f$ and $t$; similarly, $\perp$ and $T$ will refer to the minimum and maximum of the knowledge lattice.

Convention 1.1 (Depicting pre-bilattices)

It is well-known that all finite lattices (indeed, all partial orders) can be depicted in the Euclidean plane as Hasse diagrams, and it is common to represent the involved order in the vertical axis. The upward line segments depicted in a Hasse diagram correspond to the covering relation associated with the partial order. We remind the reader that the covering relation $<^{1}$ associated with a partial order $\leq$ is the strict order (i.e., irreflexive and transitive) such that $x<^{1} y$ holds iff $y$ is an immediate successor of $x$. So the order given by a Hasse diagram is the reflexive transitive closure of the

${ }^{1}$ This was already pointed out in $[29$, p. 111]. 


\section{The logic of distributive bilattices}

upward line segments depicted in the Hasse diagram. In the literature on bilattices it is common to adopt a similar convention for depicting some bilattices, but as far as the authors are aware this convention has not been explicitly stated ${ }^{2}$. Under this convention, the elements of a bilattice are represented by points of the Euclidean plane and the diagram representing the bilattice connects two different points $x$ and $y$ by a

(rep1): horizontal-rightward line segment (rep2): upward-vertical line segment (rep3): upward-rightward line segment (rep4): upward-leftward line segment

$\begin{array}{ll}\text { iff } & x<_{t}^{1} y \text { and } x \mathbb{Z}_{k} y \text { and } x \geq_{k} y, \\ \text { iff } & x<_{k}^{1} y \text { and } x \mathbb{Z}_{t} y \text { and } x \geq_{t} y, \\ \text { iff } & \langle x, y\rangle \in\left(\leq_{k} \cap \leq_{t}\right) \cap\left(<_{k}^{1} \cup<_{t}^{1}\right), \\ \text { iff } & \langle x, y\rangle \in\left(\leq_{k} \cap \geq_{t}\right) \cap\left(<_{k}^{1} \cup>_{t}^{1}\right) .\end{array}$

Hence, the truth order is the transitive closure of the rightward line segments given in the depicted diagram, and the knowledge order is the transitive closure of the upward line segments given in the depicted diagram. The reader can find the diagrams of some pre-bilattices in Figure 1. We stress that not all finite pre-bilattices can be represented in this way. It is worth pointing out that under this convention connected points are related (in some direction) by either $<_{k}^{1}$ or $<_{t}^{1}$. However, in general it is not true ${ }^{3}$ that the upward line segments correspond to the relation $<_{k}^{1}$ and the rightward line segments correspond to the relation $<_{t}^{1}$. As a counterexample, observe that in the second pre-bilattice given in Figure 1 it holds that $\perp \Varangle_{k}^{1} \mathrm{t}$.

The literature explaining the motivation behind the two lattice orders in bilattices is quite broad. In this paper we focus on the mathematical aspects of previous definition, but as far as the motivation is concerned, let us quote the following paragraph by Fitting [19]:

The ordering $\leq_{k}$ should be thought of as ranking "degree of information". Thus if $x \leq_{k} y, y$ gives us at least as much information as $x$ (and possibly more). I suppose this really should be written as $\leq_{i}$, using $i$ for information instead of $k$ for knowledge. In some papers in the literature $i$ is used, but I have always written $\leq_{k}$, and now I'm stuck with it.

Following Fitting and the tradition, we also adopt the notation $\leq_{k}$, but we agree that $\leq_{i}$ might have been a better choice.

Of course the interest in pre-bilattices increases when there is some connection between the orders. Such a connection has been introduced in the literature in at least two different ways. A first way is to impose certain monotonicity properties: a pre-bilattice is said to be interlaced when each one of the four lattice operations $\wedge, \vee, \otimes$ and $\oplus$ is monotonic with respect to both partial orders $\leq_{t}$ and $\leq_{k}$. That is,

\footnotetext{
${ }^{2}$ The only attempt to write this down has been made by Avron [5] introducing the notion of "graphically representable" pre-bilattice (and a restricted version of it called "precisely representable"). This notion corresponds exactly to the one we introduced, restricted to conditions (rep3) and (rep4) (i.e., neither horizontal nor vertical line segments are allowed in "graphically representable" pre-bilattices). In our opinion, this definition is too restrictive: for example, the pre-billatice $\mathcal{F} \mathcal{I V \mathcal { E }}$ given in Figure 1 is not "graphically representable" in the sense of $[5]$.

${ }^{3}$ It is worth pointing out that in [5] it is proved that all interlaced bilattices can be represented in such a way. The upward line segments correspond to the relation $<_{k}^{1}$, the rightward segments correspond to $<_{t}^{1}$, and there are neither horizontal nor vertical line segments.
} 
when the quasi-equations

$$
\begin{array}{ll}
x \leq_{t} y \Rightarrow x \otimes z \leq_{t} y \otimes z & x \leq_{k} y \Rightarrow x \wedge z \leq_{k} y \wedge z \\
x \leq_{t} y \Rightarrow x \oplus z \leq_{t} y \oplus z & x \leq_{k} y \Rightarrow x \vee z \leq_{k} y \vee z
\end{array}
$$

hold. A pre-bilattice is said to be distributive when all twelve distributive laws

$$
x *(y \bullet z) \approx(x * y) \bullet(x * z) \quad \text { for every } *, \bullet \in\{\wedge, \vee, \otimes, \oplus\} .
$$

hold. We will denote, respectively, the classes of pre-bilattices, interlaced pre-bilattices and distributive pre-bilattices by PreBiLat, IntPreBiLat and DPreBiLat. It is known [6] that all these classes are equational ${ }^{4}$ and that DPreBiLat $\subseteq$ IntPreBiLat $\subseteq$ PreBiLat.

A second way to establish a link between the two lattice orders is to expand the algebraic language with a new connective. This is the method originally used by Ginsberg to introduce bilattices. A bilattice is an algebra $\mathbf{B}=\langle B, \wedge, \vee, \otimes, \oplus, \neg\rangle$ such that the reduct $\langle B, \wedge, \vee, \otimes, \oplus\rangle$ is a pre-bilattice and the negation $\neg$ is a unary operation satisfying that for every $a, b \in B$,

(bil1): if $a \leq_{t} b$, then $\neg b \leq_{t} \neg a$,

(bil2): if $a \leq_{k} b$, then $\neg a \leq_{k} \neg b$,

(bil3): $a=\neg \neg a$.

It is known that the following equations (De Morgan laws) hold in any bilattice

$$
\begin{array}{ll}
\neg(x \wedge y) \approx \neg x \vee \neg y & \neg(x \vee y) \approx \neg x \wedge \neg y \\
\neg(x \otimes y) \approx \neg x \otimes \neg y & \neg(x \oplus y) \approx \neg x \oplus \neg y
\end{array}
$$

and that $\neg \top=T, \neg \perp=\perp, \neg \mathrm{t}=\mathrm{f}$ and $\neg \mathrm{f}=\mathrm{t}$. It is then easy to see that the class of bilattices, denoted by BiLat, is equationally axiomatizable. Analogously to what we did in the case of pre-billatices, we will denote by IntBiLat and DBiLat the classes of interlaced bilattices and distributive bilattices, which are also equational. It is also clear that DBiLat $\subseteq \operatorname{lntBiLat} \subseteq$ BiLat.

Convention 1.2 (Depicting bilattices)

Given a finite bilattice represented as explained in Convention 1.1, we will assume that the negation is precisely the symmetry given by the vertical axis joining $\perp$ and $T$. Hence, by the involutive law the pictures corresponding to bilattices must have a vertical symmetry axis, i.e., a left-right symmetry ${ }^{5}$. It is easy to check that all pictures in Figure 1 correspond indeed to bilattices. For example, the negation in the last picture satisfies $\neg a=b$ and $\neg c=c$.

All the bilattices shown in Figure 1 have been previosly considered in the literature $[26,3]$, so we adopt the names that are more or less standard by now ${ }^{6}$. Note that we will use these names $(\mathcal{F O U \mathcal { R }}, \mathcal{F} \mathcal{I} \mathcal{V} \mathcal{E}$, etc.), except where otherwise stated, when they

\footnotetext{
${ }^{4}$ Pre-bilattices in [6] are always bounded in both orders, but the reader can easily check that the proofs given there for these results do not use this fact. However, this is not the case for the representation theorem [6, Theorem $3.3]$, that uses crucially the boundedness assumption.

${ }^{5}$ Fitting [18] also studied the case of a connective corresponding to a top-bottom symmetry, that he called conflation.

${ }^{6}$ In the rest of cases we will use boldface roman letters $\mathbf{A}, \mathbf{B}, \mathbf{C}, \ldots, \mathbf{F m}$ to denote algebras.
} 


\section{The logic of distributive bilattices}

are viewed as bilattices (so endowed with a negation). It is known [3] that $\mathcal{F O U R}$ and $\mathcal{N} \mathcal{I N \mathcal { E }}$ are distributive bilattices (hence interlaced), while neither $\mathcal{F} \mathcal{I V E}$ nor $\mathcal{D E} \mathcal{F} \mathcal{A} \mathcal{U} \mathcal{L}$ are interlaced.

The smallest non-trivial bilattice is the four-element algebra $\mathcal{F O U R}$ depicted in Figure 1. $\mathcal{F O U R}$ is distributive and it is a simple algebra (this follows from cardinal minimality). As pointed out in [3], the role of $\mathcal{F O U \mathcal { R }}$ is a special one among bilattices, analogous to the role played among Boolean algebras by the two-element one. Let us note that the $\{\wedge, \vee, \neg\}$-reduct of $\mathcal{F O U R}$ is the four-element De Morgan lattice that is known to generate the variety of De Morgan lattices [20]. Indeed, the Belnap-Dunn four-valued logic is the logic determined by the logical matrix $\langle\mathbf{A}, \operatorname{Tr}\rangle$ where $\mathbf{A}$ is this four-element Morgan lattice and $\operatorname{Tr}=\{T, \mathrm{t}\}$ [20, Proposition 2.3]. According to the interpretation proposed by Belnap and Dunn for their logic $[7,8,1]$, which has become standard, the elements of $\mathcal{F O U R}$ may be thought of as only true (t), only false (f), both true and false $(\top)$, and neither true nor false $(\perp)$. Thus, it is reasonable to take as set of designated elements those values which are at least true (but possibly also false), that is, to take $\operatorname{Tr}=\{T, \mathrm{t}\}$. This is what Arieli and Avron [3] did when they introduced the logic $\mathcal{L B}$ into the realm of bilattices.

DEFINITION 1.3

$\mathcal{L B}=\left\langle\mathbf{F m}, \vDash_{\mathcal{L B}}\right\rangle$ is the logic determined by the logical matrix $\langle\mathcal{F} \mathcal{O U} \mathcal{R}, \operatorname{Tr}\rangle$.

The algebra $\mathbf{F m}$ of formulas is the free algebra generated by a countable set $\mathcal{V} a r$ of variables using the algebraic language $\{\wedge, \vee, \otimes, \oplus, \neg\}$. Note that there are no constants in the language. By definition, for every set $\Gamma \cup\{\varphi\}$ of formulas it holds that

- $\Gamma \vDash_{\mathcal{L B}} \varphi$, iff

- for every valuation $h \in \operatorname{Hom}(\mathbf{F m}, \mathcal{F O U R})$, if $h[\Gamma] \subseteq \operatorname{Tr}$ then $h(\varphi) \in \operatorname{Tr}$.

The study of $\mathcal{L B}$ was already started in [3]; to close this section we will remind two important results obtained there. The first is the introduction of a complete axiomatization by means of a sequent calculus. By a sequent we mean a pair $\langle\Gamma, \Delta\rangle$ where $\Gamma$ and $\Delta$ are both finite non-empty sets of formulas; we will denote the sequent $\langle\Gamma, \Delta\rangle$ by $\Gamma \triangleright \varphi$ in order to avoid any misunderstanding with other symbols that are sometimes used as sequent separator, such as $\vdash, \rightarrow$ or $\Rightarrow$. The Gentzen system introduced in [3] is ${ }^{7}$ the one defined by the axioms and rules given in Table 1, which we call $\mathcal{G}_{\mathcal{L B}}$. Since the left-hand and right-hand side of our sequents are (finite) sets of formulas, rather than multisets or sequences, it is not necessary to include the structural rules of contraction and exchange, as they are, so to speak, built-in in the formalism. Note also that, using $(\mathrm{Ax})$, Cut, $(\wedge \triangleright)$ and $(\triangleright \vee)$, it is easy to prove that the sequent $\Gamma \triangleright \Delta$ is equivalent to $\bigwedge \Gamma \triangleright \bigvee \Delta$. Taking this into account, we may obtain formal proofs of the rules of left weakening $(W \triangleright)$ and right weakening $(\triangleright W)$, as follows:

$$
(C u t) \frac{\frac{(A x)}{\bigwedge \Gamma, \varphi \triangleright \wedge \Gamma} \frac{\Gamma \triangleright \Delta}{\Lambda \Gamma \triangleright \bigvee \Delta}}{\frac{\bigwedge \Gamma, \varphi \triangleright \bigvee \Delta}{\Gamma, \varphi \triangleright \Delta}} \quad \frac{\frac{\Gamma \triangleright \Delta}{\Lambda \Gamma \triangleright \bigvee \Delta} \frac{(A x)}{\bigvee \Delta \triangleright \bigvee \Delta, \varphi}}{\frac{\Lambda \Gamma \triangleright \bigvee \Delta, \varphi}{\Gamma \triangleright \Delta, \varphi}}(\text { Cut })
$$

\footnotetext{
${ }^{7}$ Note that our presentation requires that both sides of sequents be non-empty. However, it is straightforward to
} see that the two presentations generate essentially the same consequence relation. 
Axiom: $\quad(A x) \quad \Gamma, \varphi \triangleright \varphi, \Delta$.

Rules: Cut Rule plus the following logical rules.

$$
\begin{aligned}
& (\wedge \triangleright) \frac{\Gamma, \varphi, \psi \triangleright \Delta}{\Gamma, \varphi \wedge \psi \triangleright \Delta} \\
& (\neg \wedge \triangleright) \quad \frac{\Gamma, \neg \varphi \triangleright \Delta \quad \Gamma, \neg \psi \triangleright \Delta}{\Gamma, \neg(\varphi \wedge \psi) \triangleright \Delta} \quad(\triangleright \neg \wedge) \quad \frac{\Gamma \triangleright \Delta, \neg \varphi, \neg \psi}{\Gamma \triangleright \Delta, \neg(\varphi \wedge \psi)} \\
& (\vee \triangleright) \frac{\Gamma, \varphi \triangleright \Delta \Gamma, \psi \triangleright \Delta}{\Gamma, \varphi \vee \psi \triangleright \Delta} \quad(\triangleright \vee) \frac{\Gamma \triangleright \Delta, \varphi, \psi}{\Gamma \triangleright \Delta, \varphi \vee \psi} \\
& (\neg \vee \triangleright) \quad \frac{\Gamma, \neg \varphi, \neg \psi \triangleright \Delta}{\Gamma, \neg(\varphi \vee \psi) \triangleright \Delta} \quad(\triangleright \neg \vee) \quad \frac{\Gamma \triangleright \Delta, \neg \varphi \quad \Gamma \triangleright \Delta, \neg \psi}{\Gamma \triangleright \Delta, \neg(\varphi \vee \psi)} \\
& (\otimes \triangleright) \frac{\Gamma, \varphi, \psi \triangleright \Delta}{\Gamma, \varphi \otimes \psi \triangleright \Delta} \quad(\triangleright \otimes) \frac{\Gamma \triangleright \Delta, \varphi \quad \Gamma \triangleright \Delta, \psi}{\Gamma \triangleright \Delta, \varphi \otimes \psi} \\
& (\neg \otimes \triangleright) \quad \frac{\Gamma, \neg \varphi, \neg \psi \triangleright \Delta}{\Gamma, \neg(\varphi \otimes \psi) \triangleright \Delta} \quad(\triangleright \neg \otimes) \quad \frac{\Gamma \triangleright \Delta, \neg \varphi \quad \Gamma \triangleright \Delta, \neg \psi}{\Gamma \triangleright \Delta, \neg(\varphi \otimes \psi)} \\
& (\oplus \triangleright) \frac{\Gamma, \varphi \triangleright \Delta \Gamma, \psi \triangleright \Delta}{\Gamma, \varphi \oplus \psi \triangleright \Delta} \quad(\triangleright \oplus) \frac{\Gamma \triangleright \Delta, \varphi, \psi}{\Gamma \triangleright \Delta, \varphi \oplus \psi} \\
& (\neg \oplus \triangleright) \quad \frac{\Gamma, \neg \varphi \triangleright \Delta \quad \Gamma, \neg \psi \triangleright \Delta}{\Gamma, \neg(\varphi \oplus \psi) \triangleright \Delta} \quad(\triangleright \neg \oplus) \quad \frac{\Gamma \triangleright \Delta, \neg \varphi, \neg \psi}{\Gamma \triangleright \Delta, \neg(\varphi \oplus \psi)} \\
& (\neg \neg \triangleright) \frac{\Gamma, \varphi \triangleright \Delta}{\Gamma, \neg \neg \varphi \triangleright \Delta} \quad(\triangleright \neg \neg) \frac{\Gamma \triangleright \Delta, \varphi}{\Gamma \triangleright \Delta, \neg \neg \varphi}
\end{aligned}
$$

TABLE 1. A complete sequent calculus for the logic $\mathcal{L B}$

Therefore all structural rules hold in this calculus. [3] contains a proof that this calculus admits Cut Elimination (i.e., the Cut Rule is admissible) and the following completeness result [3, Theorem 3.7$]$ :

\section{THEOREM 1.4}

The calculus $\mathcal{G}_{\mathcal{L B}}$ is complete with respect to $\vDash_{\mathcal{L B}}$. That is, $\Gamma \vDash_{\mathcal{L B}} \varphi$ iff the sequent $\Gamma \triangleright \varphi$ is derivable, without any assumption, in $\mathcal{G}_{\mathcal{L B}}$.

The second result from [3] we want to recall is the one that explains why $\mathcal{L B}$ is called the logic of logical bilattices, and amounts to saying that in order to define $\vDash_{\mathcal{L B}}$ we can replace the logical matrix $\langle\mathcal{F} \mathcal{O U} \mathcal{R}, \operatorname{Tr}\rangle$ by many other matrices.

Let $\mathbf{B}$ be a bilattice. A bifilter of $\mathbf{B}$ is a non-empty set $F \subseteq B$ such that it is a lattice filter of both orders $\leq_{t}$ and $\leq_{k}$. That is, $F$ is a subset such that, for every $a, b \in B$,

(bifilter): $a \wedge b \in F \quad$ iff $\quad a \in F$ and $b \in F \quad$ iff $\quad a \otimes b \in F$.

A prime bifilter is a proper (i.e. $F \subsetneq B$ ) bifilter such that, for every $a, b \in B$,

(prime): $a \vee b \in F \quad$ iff $\quad a \in F$ or $b \in F \quad$ iff $\quad a \oplus b \in F$.

$\mathcal{F O U R}$ only has one non-trivial bifilter, i.e. $\operatorname{Tr}$, which is also prime. The notion of logical bilattice, introduced in [3], denotes a pair $\langle\mathbf{B}, F\rangle$ where $\mathbf{B}$ is a bilattice and $F$ 


\section{The logic of distributive bilattices}

is a prime bifilter of $\mathbf{B}$. It is obvious that logical bilattices are also logical matrices in the sense of AAL, so each logical bilattice determines a logic. The second key result from [3] is then that all logical bilattices define the same logic, namely $\vDash_{\mathcal{L B}}$.

THEOREM 1.5

For any logical bilattice $\langle\mathbf{B}, F\rangle$, the logic determined by the matrix $\langle\mathbf{B}, F\rangle$ coincides with $\vDash_{\mathcal{L B}}$. That is, for every set $\Gamma \cup\{\varphi\}$ of formulas

$$
\Gamma \vDash_{\mathcal{L B}} \varphi \quad \text { iff } \quad \Gamma \models_{\langle\mathbf{B}, F\rangle} \varphi .
$$

The previous theorem is indeed a straightforward consequence of the following result (cf. [3, Theorem 2.17]):

LEMMA 1.6

Let $\mathbf{B} \in$ BiLat and $F \subsetneq B$. Then the following statements are equivalent:

1. $F$ is a prime bifilter of $\mathbf{B}$

2. There is a unique epimorphism $\pi_{F}: \mathbf{B} \longrightarrow \mathcal{F O U R}$ such that $F=\pi_{F}^{-1}[\operatorname{Tr}]$

3. There is an epimorphism $\pi_{F}: \mathbf{B} \longrightarrow \mathcal{F O U \mathcal { R }}$ such that $F=\pi_{F}^{-1}[\operatorname{Tr}]$.

It may be interesting to point out that the epimorhism $\pi_{F}$ is the map defined by

$$
\pi_{F}(b):= \begin{cases}\top & \text { if } b \in F \text { and } \neg b \in F \\ \mathrm{t} & \text { if } b \in F \text { and } \neg b \notin F \\ \mathrm{f} & \text { if } b \notin F \text { and } \neg b \in F \\ \perp & \text { if } b \notin F \text { and } \neg b \notin F .\end{cases}
$$




\begin{tabular}{|ccc|}
\hline $\mathrm{R} 1) \frac{p \wedge q}{p}$ & $(\mathrm{R} 2) \frac{p \wedge q}{q}$ & $(\mathrm{R} 3) \frac{p}{p \wedge q}$ \\
$(\mathrm{R} 4) \frac{p}{p \vee q}$ & $(\mathrm{R} 5) \frac{p \vee q}{q \vee p}$ & $(\mathrm{R} 6) \frac{p \vee p}{p}$ \\
$(\mathrm{R} 7) \frac{p \vee(q \vee r)}{(p \vee q) \vee r}$ & $(\mathrm{R} 8) \frac{p \vee(q \wedge r)}{(p \vee q) \wedge(p \vee r)}$ & $(\mathrm{R} 9) \frac{(p \vee q) \wedge(p \vee r)}{p \vee(q \wedge r)}$ \\
$(\mathrm{R} 10) \frac{p \vee r}{\neg \neg p \vee r}$ & $(\mathrm{R} 11) \frac{\neg \neg p \vee r}{p \vee r}$ & $(\mathrm{R} 12) \frac{\neg(p \vee q) \vee r}{(\neg p \wedge \neg q) \vee r}$ \\
$(\mathrm{R} 13) \frac{(\neg p \wedge \neg q) \vee r}{\neg(p \vee q) \vee r}$ & $(\mathrm{R} 14) \frac{\neg(p \wedge q) \vee r}{(\neg p \vee \neg q) \vee r}$ & $(\mathrm{R} 15) \frac{(\neg p \vee \neg q) \vee r}{\neg(p \wedge q) \vee r}$ \\
$(\mathrm{R} 16) \frac{(p \otimes q) \vee r}{(p \wedge q) \vee r}$ & $(\mathrm{R} 17) \frac{(p \wedge q) \vee r}{(p \otimes q) \vee r}$ & $(\mathrm{R} 18) \frac{(p \oplus q) \vee r}{(p \vee q) \vee r}$ \\
$(\mathrm{R} 19) \frac{(p \vee q) \vee r}{(p \oplus q) \vee r}$ & $(\mathrm{R} 20) \frac{(\neg p \otimes \neg q) \vee r}{\neg(p \otimes q) \vee r}$ & $(\mathrm{R} 21) \frac{\neg(p \otimes q) \vee r}{(\neg p \otimes \neg q) \vee r}$ \\
$(\mathrm{R} 22) \frac{(\neg p \oplus \neg q) \vee r}{\neg(p \oplus q) \vee r}$ & $(\mathrm{R} 23) \frac{\neg(p \oplus q) \vee r}{(\neg p \oplus \neg q) \vee r}$ & \\
\hline
\end{tabular}

TABLE 2. A complete Hilbert-style calculus for the logic $\mathcal{L B}$

\section{Hilbert-style presentation}

So far in the literature no Hilbert-style presentation for the logic $\mathcal{L B}$ of logical bilattices has beed given. The aim of this section is to fill in this gap by introducing a strongly complete Hilbert-style calculus for this logic. Although from a proof theoretic point of view sequent calculi (especially the ones enjoying cut elimination and the subformula property) are better suited for searching proofs than Hilbert-style ones, this is just the opposite from the point of view of AAL. The reason is that using a Hilbert-style presentation it is quite easy to characterize, in any algebraic model of a logic, the sets of elements that are closed under the rules of the logic (i.e., the filters of the logic $[23,22])$. In other words, from the AAL point of view the fact of having a Hilbert-style presentation means a lot of benefits.

Using the fact that $\{\perp\}$ is a subalgebra of $\mathcal{F O U R}$, it is easy to see that $\mathcal{L B}$ has no theorems: this is so because the map that assigns $\perp$ to all variables is a homomorphism, so there can be no formula that takes a designated value (i.e. $\mathrm{t}$ or $T$ ) for any valuation ${ }^{8}$. Therefore, all Hilbert-style presentations for $\mathcal{L B}$ have to be free of axioms and based only on (proper) rules. But as noted in [20], and contrary to what is claimed in [3, p. 37], this absence of theorems does not mean that there can be no

${ }^{8}$ Here it is crucial that we do not have any of the constants $T, t, f$ in the language, because otherwise $\perp$ would no longer be a subalgebra of $\mathcal{F O U \mathcal { R }}$. 


\section{The logic of distributive bilattices}

Hilbert-style presentation for $\mathcal{L B}$.

By the semantical definition of $\mathcal{L B}$ it is obvious that this logic is a conservative expansion of the Belnap-Dunn four-valued logic. Hence, in order to present a Hilbertstyle presentation for $\mathcal{L B}$ we can try to expand any known Hilbert-style axiomatization for the Belnap-Dunn logic. An example of such axiomatization is the calculus introduced by Font [20], consisting in the first fifteen rules of Table 2 .

DEFINITION 2.1

The logic $\vdash_{H}$ is the consequence relation defined through the rules (there are no axioms) stated in Table 2. The closure operator associated with $\vdash_{H}$ will be denoted by $\mathbf{C}_{H}$.

In the rest of the section we will prove that this calculus is strongly complete w.r.t. the semantics of $\mathcal{L B}$. The strategy of our proof is very similar to the one used in [20] for the Belnap-Dunn logic, and is based on a normal form representation of formulas.

Proposition 2.2 (Soundness)

Given a set of formulas $\Gamma \subseteq F m$ and a formula $\varphi \in F m$, if $\Gamma \vdash_{H} \varphi$, then $\Gamma \vDash_{\mathcal{L B}} \varphi$.

Proof. It is sufficient to check that the set $\operatorname{Tr}$ is closed w.r.t. all the rules given in Table 2.

Proposition 2.3

The following rules follow from (R1) to (R23):

(a) The rule $\left(\mathrm{Ri}^{+}\right) \frac{\varphi}{\psi}$, for each one of the rules $(\mathrm{Ri}) \frac{\varphi \vee r}{\psi \vee r}$ (where $\left.i \in\{10, \ldots, 23\}\right)$.

(b) The rule $\frac{\varphi \wedge r}{\psi \wedge r}$ in the same cases.

Proof. (a) From $\varphi$ by (R4) we obtain $\varphi \vee \psi$. Then we apply (Ri) to get $\psi \vee \psi$ and by (R6) we obtain $\psi$.

(b) From $\varphi \wedge r$ by (R1) we obtain $\varphi$. Now using (a) we get $\psi$. Also from $\varphi \wedge r$, by (R2), follows $r$. Thus applying (R3) we get $\psi \wedge r$.

Proposition 2.4

From $(\mathrm{R} 1), \ldots,(\mathrm{R} 9)$ and $\left(\mathrm{R} 16^{+}\right), \ldots,\left(\mathrm{R} 19^{+}\right)$we can easily derive the following rules:
(R1') $\frac{p \otimes q}{p}$
(R2') $\frac{p \otimes q}{q}$
(R3') $\frac{p \quad q}{p \otimes q}$
(R4') $\frac{p}{p \oplus q}$
(R5') $\frac{p \oplus q}{q \oplus p}$
(R6') $\frac{p \oplus p}{p}$
(R7') $\frac{p \oplus(q \oplus r)}{(p \oplus q) \oplus r}$
(R8') $\frac{p \oplus(q \otimes r)}{(p \oplus q) \otimes(p \oplus r)}$
(R9') $\frac{(p \oplus q) \otimes(p \oplus r)}{p \oplus(q \otimes r)}$

Proposition 2.5

The interderivability relation $\nvdash_{H}$ is a congruence w.r.t. the operations $\wedge$ and $\vee$. 
Proof. It is sufficient to show that the following two rules

$$
\frac{p \wedge r \quad q \wedge r}{(p \wedge q) \wedge r} \quad \frac{p \vee r \quad q \vee r}{(p \wedge q) \vee r}
$$

together with the rules $\frac{\varphi \vee r}{\psi \vee r}$ and $\frac{\varphi \wedge r}{\psi \wedge r}$ (for each rule $\frac{\varphi}{\psi}$ in Table 2$)$ are all of them derivable in $\vdash_{H}$. For the rules in Table 2 that belong to the $\langle\wedge, \vee\rangle$ fragment it is known that they follow just from rules (R1) to (R9). And for (R10) to (R23) the conjunction case is shown by Proposition 2.3(b), while the disjunction case can be easily shown by using the associativity of $\vee$.

DEFINITION 2.6

$\mathcal{L}$ it $=\mathcal{V} a r \cup\{\neg p: p \in \mathcal{V} a r\}$ is the set of literals. $\mathcal{C} l$, the set of clauses, is the least set containing $\mathcal{L}$ it and closed under $\vee$. For any $\varphi \in F m$, the set var $(\varphi)$ of variables of $\varphi$ is defined in the usual way; for $\Gamma \subseteq F m, \operatorname{var}(\Gamma)=\bigcup_{\varphi \in \Gamma} \operatorname{var}(\varphi)$. For any $\varphi \in \mathcal{C} l$, the set lit $(\varphi)$ of literals of $\varphi$ is defined inductively by $\operatorname{lit}(\varphi)=\{\varphi\}$ if $\varphi \in \mathcal{L}$ it and $\operatorname{lit}(\varphi \vee \psi)=\operatorname{lit}(\varphi) \cup \operatorname{lit}(\psi)$. For $\Gamma \subseteq \mathcal{C} l, \operatorname{lit}(\Gamma)=\bigcup_{\varphi \in \Gamma} \operatorname{lit}(\varphi)$.

Proposition 2.7

For all $\varphi \in F m$ there is a finite $\Gamma \subseteq \mathcal{C l}$ such that $\operatorname{var}(\varphi)=\operatorname{var}(\Gamma)$ and for every $\psi \in F m, \mathbf{C}_{H}(\varphi \vee \psi)=\mathbf{C}_{H}(\{\gamma \vee \psi: \gamma \in \Gamma\})$.

ProOF. By induction on the length of $\varphi$.

If $\varphi=p \in \mathcal{V}$ ar then $\Gamma=\{p\}$.

If $\varphi=\varphi_{1} \wedge \varphi_{2}$ and by inductive hypothesis $\Gamma_{1}, \Gamma_{2}$ correspond respectively to $\varphi_{1}$ and $\varphi_{2}$ then we may take $\Gamma=\Gamma_{1} \cup \Gamma_{2}$ and we have $\operatorname{var}(\varphi)=\operatorname{var}(\Gamma)$. We also have

$$
\begin{aligned}
\mathbf{C}_{H}(\varphi \vee \psi) & =\mathbf{C}_{H}\left(\left(\varphi_{1} \wedge \varphi_{2}\right) \vee \psi\right) \\
& =\mathbf{C}_{H}\left(\left(\varphi_{1} \vee \psi\right) \wedge\left(\varphi_{2} \vee \psi\right)\right) \\
& =\mathbf{C}_{H}\left(\varphi_{1} \vee \psi, \varphi_{2} \vee \psi\right) \\
& =\operatorname{by}(\mathrm{R} 1),(\mathrm{R} 2),(\mathrm{R} 3) \\
& =\mathbf{C}_{H}\left(\mathbf{C}_{H}\left(\varphi_{1} \vee \psi\right) \cup \mathbf{C}_{H}\left(\varphi_{2} \vee \psi\right)\right) \\
& =\mathbf{C}_{H}\left(\mathbf{C}_{H}\left(\left\{\gamma_{1} \vee \psi: \gamma_{1} \in \Gamma_{1}\right\}\right) \cup \mathbf{C}_{H}\left(\left\{\gamma_{2} \vee \psi: \gamma_{2} \in \Gamma_{2}\right\}\right)\right) \\
& =\mathbf{C}_{H}(\{\gamma \vee \psi: \gamma \in \Gamma\}) .
\end{aligned}
$$

If $\varphi=\varphi_{1} \vee \varphi_{2}$ and $\Gamma_{1}, \Gamma_{2}$ correspond respectively to $\varphi_{1}$ and $\varphi_{2}$ then take $\Gamma=$ $\left\{\gamma_{1} \vee \gamma_{2}: \gamma_{1} \in \Gamma_{1}, \gamma_{2} \in \Gamma_{2}\right\}$ and we have $\operatorname{var}(\varphi)=\operatorname{var}(\Gamma)$. We also have:

$$
\begin{aligned}
\mathbf{C}_{H}(\varphi \vee \psi) & =\mathbf{C}_{H}\left(\left(\varphi_{1} \vee \varphi_{2}\right) \vee \psi\right) \\
& =\mathbf{C}_{H}\left(\varphi_{1} \vee\left(\varphi_{2} \vee \psi\right)\right) \\
& =\text { (by inductive hypothesis }) \\
& =\mathbf{C}_{H}\left(\left\{\gamma_{1} \vee\left(\varphi_{2} \vee \psi\right): \gamma_{1} \in \Gamma_{1}\right\}\right) \\
& =\mathbf{C}_{H}\left(\left\{\varphi_{2} \vee\left(\gamma_{1} \vee \psi\right): \gamma_{1} \in \Gamma_{1}\right\}\right) \\
& =\mathbf{C}_{H}\left(\left\{\gamma_{2} \vee\left(\gamma_{1} \vee \psi\right): \gamma_{1} \in \Gamma_{1}, \gamma_{2} \in \Gamma_{2}\right\}\right) \\
& =\mathbf{C}_{H}\left(\left\{\left(\gamma_{1} \vee \gamma_{2}\right) \vee \psi: \gamma_{1} \in \Gamma_{1}, \gamma_{2} \in \Gamma_{2}\right\}\right)
\end{aligned}
$$


12 The logic of distributive bilattices

If $\varphi=\varphi_{1} \otimes \varphi_{2}$ then $\mathbf{C}_{H}(\varphi \vee \psi)=\mathbf{C}_{H}\left(\left(\varphi_{1} \otimes \varphi_{2}\right) \vee \psi\right)$. By (R16) and (R17) we have $\mathbf{C}_{H}\left(\left(\varphi_{1} \otimes \varphi_{2}\right) \vee \psi\right)=\mathbf{C}_{H}\left(\left(\varphi_{1} \wedge \varphi_{2}\right) \vee \psi\right)$, so we may apply the procedure for $\varphi=\varphi_{1} \wedge \varphi_{2}$.

If $\varphi=\varphi_{1} \oplus \varphi_{2}$ then $\mathbf{C}_{H}(\varphi \vee \psi)=\mathbf{C}_{H}\left(\left(\varphi_{1} \oplus \varphi_{2}\right) \vee \psi\right)$. By (R18) and (R19) we have $\mathbf{C}_{H}\left(\left(\varphi_{1} \oplus \varphi_{2}\right) \vee \psi\right)=\mathbf{C}_{H}\left(\left(\varphi_{1} \vee \varphi_{2}\right) \vee \psi\right)$, so we may apply the procedure for $\varphi=\varphi_{1} \vee \varphi_{2}$.

If $\varphi=\neg \varphi^{\prime}$ we have to distinguish several cases on $\varphi^{\prime}$.

If $\varphi^{\prime}=p \in \mathcal{V}$ ar then $\varphi \in \mathcal{L} i t \subseteq \mathcal{C} l$, so we may take $\Gamma=\{\varphi\}$.

If $\varphi^{\prime}=\neg \varphi^{\prime \prime}$, then $\varphi=\neg \neg \varphi^{\prime \prime}$ and by (R10) and (R11) we have $\mathbf{C}_{H}(\varphi \vee \psi)=$

$\mathbf{C}_{H}\left(\varphi^{\prime \prime} \vee \psi\right) . \varphi^{\prime \prime}$ is shorter that $\varphi$ and its corresponding set $\Gamma$ also works for $\varphi$.

If $\varphi^{\prime}=\varphi_{1} \wedge \varphi_{2}$ then $\varphi=\neg\left(\varphi_{1} \wedge \varphi_{2}\right)$ and by (R14) and (R15) we have $\mathbf{C}_{H}(\varphi \vee \psi)=$ $\mathbf{C}_{H}\left(\left(\neg \varphi_{1} \vee \neg \varphi_{2}\right) \vee \psi\right)$. Both $\neg \varphi_{1}$ and $\neg \varphi_{2}$ are shorter than $\neg\left(\varphi_{1} \wedge \varphi_{2}\right)$, so the same procedure for the case of $\varphi=\varphi_{1} \vee \varphi_{2}$ works.

If $\varphi^{\prime}=\varphi_{1} \vee \varphi_{2}$ then $\varphi=\neg\left(\varphi_{1} \vee \varphi_{2}\right)$ and by (R12) and (R13) we have $\mathbf{C}_{H}(\varphi \vee \psi)=$ $\mathbf{C}_{H}\left(\left(\neg \varphi_{1} \wedge \neg \varphi_{2}\right) \vee \psi\right)$. Both $\neg \varphi_{1}$ and $\neg \varphi_{2}$ are shorter than $\neg\left(\varphi_{1} \vee \varphi_{2}\right)$, so the same procedure for the case of $\varphi=\varphi_{1} \wedge \varphi_{2}$ works.

If $\varphi^{\prime}=\varphi_{1} \otimes \varphi_{2}$, then $\varphi=\neg\left(\varphi_{1} \otimes \varphi_{2}\right)$ and by (R20) and (R21) we have

$\mathbf{C}_{H}(\varphi \vee \psi)=\mathbf{C}_{H}\left(\left(\neg \varphi_{1} \otimes \neg \varphi_{2}\right) \vee \psi\right)$. Both $\neg \varphi_{1}$ and $\neg \varphi_{2}$ are shorter than $\neg\left(\varphi_{1} \otimes \varphi_{2}\right)$ and the procedure applied for $\varphi=\varphi_{1} \otimes \varphi_{2}$ works.

If $\varphi^{\prime}=\varphi_{1} \oplus \varphi_{2}$, then $\varphi=\neg\left(\varphi_{1} \oplus \varphi_{2}\right)$ and by (R22) and (R23) we have $\mathbf{C}_{H}(\varphi \vee \psi)=\mathbf{C}_{H}\left(\left(\neg \varphi_{1} \oplus \neg \varphi_{2}\right) \vee \psi\right)$. Both $\neg \varphi_{1}$ and $\neg \varphi_{2}$ are shorter than $\neg\left(\varphi_{1} \oplus \varphi_{2}\right)$ and again the procedure applied for $\varphi=\varphi_{1} \oplus \varphi_{2}$ works.

Proposition 2.8

For all $\varphi \in F m$ there is a finite $\Gamma_{\varphi} \subseteq \mathcal{C} l$ such that $\operatorname{var}(\varphi)=\operatorname{var}\left(\Gamma_{\varphi}\right)$ and $\mathbf{C}_{H}(\varphi)=$ $\mathbf{C}_{H}\left(\Gamma_{\varphi}\right)$.

Proof. By induction on the length of $\varphi$.

If $\varphi=p \in \mathcal{V}$ ar, then take $\Gamma_{\varphi}=\{\varphi\}$.

If $\varphi=\varphi_{1} \wedge \varphi_{2}$ by (R1), (R2) and (R3) we have $\mathbf{C}_{H}(\varphi)=\mathbf{C}_{H}\left(\varphi_{1}, \varphi_{2}\right)$. So we may take $\Gamma_{\varphi}=\Gamma_{\varphi_{1}} \cup \Gamma_{\varphi_{2}}$ and we are done.

If $\varphi=\varphi_{1} \vee \varphi_{2}$ then by Proposition 2.7 and (R5) we have:

$$
\begin{aligned}
\mathbf{C}_{H}(\varphi) & =\mathbf{C}_{H}\left(\left\{\gamma_{1} \vee \varphi_{2}: \gamma_{1} \in \Gamma_{1}\right\}\right) \\
& =\mathbf{C}_{H}\left(\left\{\varphi_{2} \vee \gamma_{1}: \gamma_{1} \in \Gamma_{1}\right\}\right) \\
& =\mathbf{C}_{H}\left(\left\{\gamma_{2} \vee \gamma_{1}: \gamma_{1} \in \Gamma_{1}, \gamma_{2} \in \Gamma_{2}\right\}\right)
\end{aligned}
$$

Since $\Gamma_{1}, \Gamma_{2} \subseteq \mathcal{C l}$ are finite, $\Gamma_{\varphi}=\left\{\gamma_{1} \vee \gamma_{2}: \gamma_{1} \in \Gamma_{1}, \gamma_{2} \in \Gamma_{2}\right\} \subseteq \mathcal{C l}$ is also finite and we are done.

If $\varphi=\varphi_{1} \otimes \varphi_{2}$, by $\left(\mathrm{R} 16^{+}\right)$and $\left(\mathrm{R} 17^{+}\right)$we have $\mathbf{C}_{H}(\varphi)=\mathbf{C}_{H}\left(\varphi_{1}, \varphi_{2}\right)$, so we may take $\Gamma_{\varphi}=\Gamma_{\varphi_{1}} \cup \Gamma_{\varphi_{2}}$ and we are done.

If $\varphi=\varphi_{1} \oplus \varphi_{2}$, since by $\left(\mathrm{R} 18^{+}\right)$and $\left(\mathrm{R} 19^{+}\right)$we have $\mathbf{C}_{H}\left(\varphi_{1} \oplus \varphi_{2}\right)=\mathbf{C}_{H}\left(\varphi_{1} \vee \varphi_{2}\right)$, we may apply the procedure for $\varphi=\varphi_{1} \vee \varphi_{2}$.

If $\varphi=\neg \varphi^{\prime}$ we have to distinguish several cases.

If $\varphi^{\prime}=p \in \mathcal{V} a r$, then $\varphi \in \mathcal{C} l$, so we may take $\Gamma_{\varphi}=\{\varphi\}$. 
If $\varphi^{\prime}=\neg \varphi^{\prime \prime}$, then by $\left(\mathrm{R} 10^{+}\right)$and $\left(\mathrm{R} 11^{+}\right)$we have $\mathbf{C}_{H}(\varphi)=\mathbf{C}_{H}\left(\varphi^{\prime \prime}\right)$ and since $\varphi^{\prime \prime}$ is shorter that $\varphi$ we are done.

If $\varphi^{\prime}=\varphi_{1} \wedge \varphi_{2}$ then by $\left(\mathrm{R} 14^{+}\right)$and $\left(\mathrm{R} 15^{+}\right)$we have $\mathbf{C}_{H}(\varphi)=\mathbf{C}_{H}\left(\neg \varphi_{1} \vee \neg \varphi_{2}\right)$, so we may apply the procedure for $\varphi=\varphi_{1} \vee \varphi_{2}$.

If $\varphi^{\prime}=\varphi_{1} \vee \varphi_{2}$ then by $\left(\mathrm{R} 12^{+}\right)$and $\left(\mathrm{R} 13^{+}\right)$we have $\mathbf{C}_{H}(\varphi)=\mathbf{C}_{H}\left(\neg \varphi_{1}, \neg \varphi_{2}\right)$, so applying the inductive hypothesis we are done.

If $\varphi^{\prime}=\varphi_{1} \otimes \varphi_{2}$, then $\varphi=\neg\left(\varphi_{1} \otimes \varphi_{2}\right)$ and by $\left(\mathrm{R} 20^{+}\right)$and $\left(\mathrm{R} 21^{+}\right)$we have

$\mathbf{C}_{H}(\varphi)=\mathbf{C}_{H}\left(\neg \varphi_{1} \otimes \neg \varphi_{2}\right)$, so the procedure applied for $\varphi=\varphi_{1} \otimes \varphi_{2}$ works.

If $\varphi^{\prime}=\varphi_{1} \oplus \varphi_{2}$, then $\varphi=\neg\left(\varphi_{1} \oplus \varphi_{2}\right)$ and by $\left(\mathrm{R} 22^{+}\right)$and $\left(\mathrm{R}^{2} 3^{+}\right)$we have

$\mathbf{C}_{H}(\varphi)=\mathbf{C}_{H}\left(\neg \varphi_{1} \oplus \neg \varphi_{2}\right)$, so the procedure applied for $\varphi=\varphi_{1} \oplus \varphi_{2}$ works.

\section{ThEOREM 2.9 (Normal Form)}

Every formula is equivalent, both through $\nvdash_{H}$ and $=\models_{\mathcal{L B}}$, to a $\wedge$-conjunction of clauses with the same variables.

Proof. By Proposition 2.8 we have that $\varphi-\Vdash_{H} \wedge \Gamma_{\varphi}$, where $\bigwedge \Gamma_{\varphi}$ is any conjunction of all the clauses in $\Gamma_{\varphi}$. By Proposition 2.2 this implies also that $\wedge \Gamma_{\varphi}=\|_{\mathcal{L B}} \varphi$.

LEMMA 2.10

For all $\Gamma \subseteq \mathcal{C l}$ and $\varphi \in \mathcal{C l}$, the following are equivalent:

1. $\Gamma \vdash_{H} \varphi$,

2. $\Gamma \vDash_{\mathcal{L B}} \varphi$,

3. $\exists \gamma \in \Gamma$ such that $l i t(\gamma) \subseteq \operatorname{lit}(\varphi)$,

4. $\exists \gamma \in \Gamma$ such that $\gamma \vdash_{H} \varphi$.

Proof. $1 \Rightarrow 2$ follows from Proposition 2.2.

$2 \Rightarrow 3$. For a fixed $\varphi \in \mathcal{C l}$ define a homomorphism $h: \mathbf{F m} \rightarrow \mathcal{F O U R}$ as follows. For every $p \in \mathcal{V}$ ar:

$$
h(p)=\left\{\begin{aligned}
\mathrm{t} & \text { if } p \notin \operatorname{lit}(\varphi) \text { and } \neg p \in \operatorname{lit}(\varphi) \\
\top & \text { if } p, \neg p \notin \operatorname{lit}(\varphi) \\
\perp & \text { if } p, \neg p \in \operatorname{lit}(\varphi) \\
\mathrm{f} & \text { if } p \in \operatorname{lit}(\varphi) \text { and } \neg p \notin \operatorname{lit}(\varphi)
\end{aligned}\right.
$$

If $p \in \operatorname{lit}(\varphi)$ then $h(p) \in\{\mathrm{f}, \perp\}$ and also $h(\neg p) \in\{\mathrm{f}, \perp\}$ when $\neg p \in$ lit $(\varphi)$. Since $\mathrm{f} \leq_{t} \perp$, we have $h(\varphi) \in\{\mathrm{f}, \perp\}$. Suppose (1) fails: then for any $\gamma \in \Gamma$ there would be $\psi_{\gamma} \in$ lit $(\gamma)$ such that $\psi_{\gamma} \notin$ lit $(\varphi)$. Then we would have $h\left(\psi_{\gamma}\right) \in\{\mathrm{t}, \top\}$ and as a consequence $h(\gamma) \in\{\mathrm{t}, \top\}$. Thus we would have, against (2), $h[\Gamma] \subseteq\{\mathrm{t}, \top\}$ while $h(\varphi) \notin\{\mathrm{t}, \top\}$.

$3 \Rightarrow 4$. If $\operatorname{lit}(\gamma) \subseteq \operatorname{lit}(\varphi)$ and $\gamma, \varphi \in \mathcal{C l}$, then $\varphi$ is a disjunction of the same literals appearing in $\gamma$ plus other ones, modulo some associations, permutations etc. Therefore applying rules (R4) to (R7) and repeatedly using Proposition 2.5 we get $\gamma \vdash_{H} \varphi$.

$4 \Rightarrow 1$. Immediate.

Theorem 2.11 (Completeness)

For all $\Gamma \subseteq F m$ and $\varphi \in F m$, it holds that $\Gamma \vDash_{\mathcal{L B}} \varphi$ iff $\Gamma \vdash_{H} \varphi$.

Proof. By Theorem 2.9 and Lemma 2.10. 
14 The logic of distributive bilattices

\section{Algebraic study of (pre-)bilattices}

The motivation behind the birth of AAL is to establish a connection between the logic and the algebraic machineries in such a way that results from one side (logic or algebraic) can be translated into the other side. In the same spirit, the purpose of this section is to develop the algebraic apparatus behind the logic of logical bilattices, which will be used in Section 4 to study the connections between the logic $\mathcal{L B}$ and the algebraic bilattice framework. For this purpose it would be enough to focus on the class DBiLat of distributive bilattices, but for the sake of generality we will consider a broader class. We will thus obtain some algebraic results that, although not essential to the study of $\mathcal{L B}$, have in our opinion an independent interest.

\subsection{Representation theorem for interlaced pre-bilattices}

Recall that the class IntPreBiLat of interlaced pre-bilattices is formed by those prebilattices satisfying that each one of the four lattice operations is monotone with respect to both orders $\leq_{t}$ and $\leq_{k}$. This condition means that every order $\leq$ belonging to the set $\left\{\leq_{t}, \geq_{t}, \leq_{k}, \geq_{k}\right\}$ satisfies the quasi-equations

$$
x \leq y \& z \leq u \Rightarrow x * z \leq y * u \quad \text { for every } * \in\{\wedge, \vee, \otimes, \oplus\},
$$

i.e., $\leq$ is compatible with respect to all four lattice operations.

For bounded interlaced pre-bilattices there is a nice representation theorem due to Avron [6] stating that every bounded pre-bilattice can be obtained, up to isomorphism, as a particular product of two bounded lattices ${ }^{9}$. As far as the authors are aware, all published proofs of this result $[6,32,29]$ rely in an essential way on the boundedness assumption. The aim of this subsection is to prove that the theorem is also valid when we drop the boundedness assumption ${ }^{10}$. Our proof uses a new method based on the study of principal bifilters (and also filter-ideals, as we shall see) of interlaced pre-bilattices, but this connection will not be explicitly stated until Section 3.3.

\section{DEFINITION 3.1}

Let $\mathbf{L}_{1}=\left\langle L_{1}, \sqcap_{1}, \sqcup_{1}\right\rangle$ and $\mathbf{L}_{\mathbf{2}}=\left\langle L_{2}, \sqcap_{2}, \sqcup_{2}\right\rangle$ be two lattices with associated orders $\leq_{1}$ and $\leq_{2}$. Then the product pre-bilattice $\mathbf{L}_{\mathbf{1}} \odot \mathbf{L}_{\mathbf{2}}=\left\langle L_{1} \times L_{2}, \wedge, \vee, \otimes, \oplus\right\rangle$ is defined as follows. For all $\left\langle a_{1}, a_{2}\right\rangle,\left\langle b_{1}, b_{2}\right\rangle \in L_{1} \times L_{2}$,

$$
\begin{aligned}
& \left\langle a_{1}, a_{2}\right\rangle \wedge\left\langle b_{1}, b_{2}\right\rangle=\left\langle a_{1} \sqcap_{1} b_{1}, a_{2} \sqcup_{2} b_{2}\right\rangle \\
& \left\langle a_{1}, a_{2}\right\rangle \vee\left\langle b_{1}, b_{2}\right\rangle=\left\langle a_{1} \sqcup_{1} b_{1}, a_{2} \sqcap_{2} b_{2}\right\rangle \\
& \left\langle a_{1}, a_{2}\right\rangle \otimes\left\langle b_{1}, b_{2}\right\rangle=\left\langle a_{1} \sqcap_{1} b_{1}, a_{2} \sqcap_{2} b_{2}\right\rangle \\
& \left\langle a_{1}, a_{2}\right\rangle \oplus\left\langle b_{1}, b_{2}\right\rangle=\left\langle a_{1} \sqcup_{1} b_{1}, a_{2} \sqcup_{2} b_{2}\right\rangle .
\end{aligned}
$$

It easy to check that the structure $\mathbf{L}_{\mathbf{1}} \odot \mathbf{L}_{\mathbf{2}}$ is always an interlaced pre-bilattice. By the definition it is obvious that

$$
\left\langle a_{1}, a_{2}\right\rangle \leq_{k}\left\langle b_{1}, b_{1}\right\rangle \quad \text { iff } \quad a_{1} \leq_{1} a_{2} \text { and } a_{2} \leq_{2} b_{2}
$$

\footnotetext{
${ }^{9}$ For the particular case of distributive pre-bilattices this result had already been obtained by Ginsberg [26] and also by Fitting $[15,16]$.

${ }^{10}$ In the recent publication [30] it is claimed that this statement holds, but no proof is given.
} 


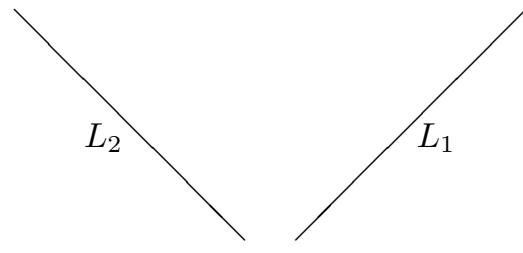

Representation of axis

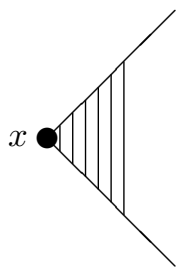

The $\leq_{t}$-upward closed The $\leq_{k}$-upward closed

FIG. 2. The cones determined by the orders in a product pre-bilattice

and

$$
\left\langle a_{1}, a_{2}\right\rangle \leq_{t}\left\langle b_{1}, b_{1}\right\rangle \quad \text { iff } \quad a_{1} \leq_{1} a_{2} \text { and } a_{2} \geq_{2} b_{2} .
$$

In Figure 2 the reader can find a "graphical representation" of the upward closed set, for each one of the orders, generated by an element $x=\left\langle a_{1}, a_{2}\right\rangle$.

It is worth pointing out that the product pre-bilattice construction is a particular case of a direct product construction. It is obvious that any lattice $\mathbf{L}=\langle L, \sqcap, \sqcup\rangle$ can be seen as a degenerated pre-bilattice in at least four different ways; we can consider the following four pre-bilattices: $\mathbf{L}^{++}:=\langle L, \sqcap, \sqcup, \sqcap, \sqcup\rangle, \mathbf{L}^{+-}:=\langle L, \sqcap, \sqcup, \sqcup, \sqcap\rangle$, $\mathbf{L}^{-+}:=\langle L, \sqcup, \sqcap, \sqcup, \sqcap\rangle$ and $\mathbf{L}^{--}:=\langle L, \sqcup, \sqcap, \sqcap, \sqcup\rangle$. The first superscript, + or - , says whether we are taking as truth order the same order than in the original lattice or the dual one; the second superscript refers to the same for the knowledge order. Using this notation it is obvious that the product pre-bilattice $\mathbf{L}_{\mathbf{1}} \odot \mathbf{L}_{\mathbf{2}}$ coincides with the direct product $\mathbf{L}_{1}^{++} \times \mathbf{L}_{\mathbf{2}}^{-+}$. We stress that $\mathbf{L}_{\mathbf{1}}^{++}=\left\langle L_{1}, \sqcap_{1}, \sqcup_{1}, \sqcap_{1}, \sqcup_{1}\right\rangle$ and $\mathbf{L}_{\mathbf{2}}^{-+}=\left\langle L_{2}, \sqcup_{2}, \sqcap_{2}, \sqcap_{2}, \sqcup_{2}\right\rangle$.

The rest of this subsection is devoted the proof of the following theorem:

THEOREM 3.2 (Representation)

Let $\mathbf{B}$ be a pre-bilattice. The following statements are equivalent.

1. $\mathbf{B}$ is an interlaced pre-bilattice.

2. There are two lattices $\mathbf{L}_{\mathbf{1}}$ and $\mathbf{L}_{\mathbf{2}}$ such that $\mathbf{B}$ is isomorphic to $\mathbf{L}_{\mathbf{1}} \odot \mathbf{L}_{\mathbf{2}}$.

For this we will need to state some more properties of interlaced pre-bilattices; but first of all let us note that there is a duality implicit in the definition of IntPreBiLat that will allow to simplify many of our proofs.

REMARK 3.3 (Duality Principle)

Let $\mathbf{B}=\left\langle B, \leq_{t}, \leq_{k}\right\rangle$ be a pre-bilattice and let $\leq_{t}^{\partial}$ and $\leq_{k}^{\partial}$ denote the dual orders of $\leq_{t}$ and $\leq_{k}$ respectively. A dual algebra of $\mathbf{B}$ is any of the pre-bilattices $\left\langle B, \leq_{t}^{\partial}, \leq_{k}\right\rangle$, $\left\langle B, \leq_{t}^{\partial}, \leq_{k}^{\partial}\right\rangle$ and $\left\langle B, \leq_{t}, \leq_{k}^{\partial}\right\rangle$. It is easy to see that the class IntPreBiLat is closed under dual algebras: hence any property that holds for all members of IntPreBiLat also holds in any dual algebra of an interlaced pre-bilattice. 


\section{The logic of distributive bilattices}

In Proposition 3.4 and Corollary 3.5 we state a result that is in our opinion crucial to understand the structure of interlaced pre-bilattices.

\section{Proposition 3.4}

Let $\mathbf{B}$ be an interlaced pre-bilattice. Then, for all $a, b \in B$,

$$
\begin{array}{rlllllll}
\text { 1. } a \leq_{k} a \wedge b & \text { iff } & \langle a, b\rangle \in \leq_{k} \circ \leq_{t} & \text { iff } & a \leq_{t} a \otimes b & \text { iff } & \langle a, b\rangle \in \leq_{t} \circ \leq_{k} & \text { iff } \\
a \vee b \leq_{k} b & \text { iff } & \langle b, a\rangle \in \geq_{k} \circ \geq_{t} & \text { iff } & a \oplus b \leq_{t} b & \text { iff } & \langle b, a\rangle \in \geq_{t} \circ \geq_{k} \text {. } & \\
\text { 2. } a \leq_{k} a \vee b & \text { iff } & \langle a, b\rangle \in \leq_{k} \circ \geq_{t} & \text { iff } & a \geq_{t} a \otimes b & \text { iff } & \langle a, b\rangle \in \geq_{t} \circ \leq_{k} & \text { iff } \\
a \wedge b \leq_{k} b & \text { iff } & \langle b, a\rangle \in \geq_{k} \circ \leq_{t} & \text { iff } & a \oplus b \geq_{t} b & \text { iff } & \langle b, a\rangle \in \leq_{t} \circ \geq_{k} . & \\
\text { 3. } a \geq_{k} a \wedge b & \text { iff } & \langle a, b\rangle \in \geq_{k} \circ \leq_{t} & \text { iff } & a \leq_{t} a \oplus b & \text { iff } & \langle a, b\rangle \in \leq_{t} \circ \geq_{k} & \text { iff } \\
a \vee b \geq_{k} b & \text { iff } & \langle b, a\rangle \in \leq_{k} \circ \geq_{t} & \text { iff } & a \otimes b \leq_{t} b & \text { iff } & \langle b, a\rangle \in \geq_{t} \circ \leq_{k} . & \\
\text { 4. } a \geq_{k} a \vee b & \text { iff } & \langle a, b\rangle \in \geq_{k} \circ \geq_{t} & \text { iff } & a \geq_{t} a \oplus b & \text { iff } & \langle a, b\rangle \in \geq_{t} \circ \geq_{k} & \text { iff } \\
a \wedge b \geq_{k} b & \text { iff } & \langle b, a\rangle \in \leq_{k} \circ \leq_{t} & \text { iff } & a \otimes b \geq_{t} b & \text { iff } & \langle b, a\rangle \in \leq_{t} \circ \leq_{k} . &
\end{array}
$$

Proof. By the Duality Principle it is enough to prove the first of these four statements. And indeed it is enough to prove the equivalences stated in the first line of this first statement (because this second line corresponds to the first line of the fourth statement just permuting $a$ and $b$ ). Let us denote by (i), (ii), (iii) and (iv) each one of the claims involved in this first line from the first statement.

(i) $\Rightarrow$ (ii): If $a \leq_{k} a \wedge b$, then it is obvious that $a \leq_{k} a \wedge b \leq_{t} b$. Therefore, $\langle a, b\rangle \in \leq_{k} \circ \leq_{t}$.

(ii) $\Rightarrow$ (iii): Let us assume that there is some $c$ such that $a \leq_{k} c \leq_{t} b$. Then, by the interlacing conditions we have $a=a \otimes c \leq_{t} a \otimes b$.

(iii) $\Rightarrow$ (iv): If $a \leq_{t} a \otimes b$, then $a \leq_{t} a \otimes b \leq_{k} b$. Thus, $\langle a, b\rangle \in \leq_{t} \circ \leq_{k}$.

(iv) $\Rightarrow$ (i): If $a \leq_{t} c \leq_{k} b$ for some $c$, then by the interlacing conditions it holds that $a=a \wedge c \leq_{k} a \wedge b$.

Corollary 3.5

Let $\mathbf{B}$ be an interlaced pre-bilattice. Then, for every $\leq_{1}, \leq_{2} \in\left\{\leq_{t}, \geq_{t}, \leq_{k}, \geq_{k}\right\}$ it holds that $\leq_{1} \circ \leq_{2}=\leq_{2} \circ \leq_{1}$.

Proof. Proposition 3.4 deals with a lot of these cases. For the rest of cases this is straightforward (note that $\leq_{t} \circ \geq_{t}=B \times B=\leq_{k} \circ \geq_{k}$ ).

An easy consequence of this corollary is that, for every $\leq_{1}, \leq_{2} \in\left\{\leq_{t}, \geq_{t}, \leq_{k}, \geq_{k}\right\}$, the relation $\leq_{1} \circ \leq_{2}$ is transitive. This is so because $\left(\leq_{1} \circ \leq_{2}\right) \circ\left(\leq_{1} \circ \leq_{2}\right)=\leq_{1}$ $\circ\left(\leq_{2} \circ \leq_{1}\right) \circ \leq_{2}=\leq_{1} \circ\left(\leq_{1} \circ \leq_{2}\right) \circ \leq_{2}=\left(\leq_{1} \circ \leq_{1}\right) \circ\left(\leq_{2} \circ \leq_{2}\right)=\leq_{1} \circ \leq_{2}$. Hence, $\leq_{1} \circ \leq_{2}$ is a quasi-order (i.e., reflexive and transitive) compatible with all four lattice operations. The compatibility trivially follows from the interlacing conditions, which say that both $\leq_{1}$ and $\leq_{2}$ are compatible with the four operations. This suggests that it may be useful to study the equivalence relation associated to these quasi-orders.

Proposition 3.6

Let $\mathbf{B}$ be an interlaced pre-bilattice. Then, for all $a, b \in B$ it holds that

$\begin{array}{llllll}\text { 1. }\langle a, b\rangle,\langle b, a\rangle \in \leq_{t} \circ \leq_{k} & \text { iff } & a \vee b \leq_{t} a \otimes b & \text { iff } & a \vee b=a \otimes b & \text { iff } \\ \langle a, b\rangle,\langle b, a\rangle \in \leq_{k} \circ \leq_{t} & \text { iff } & a \oplus b \leq_{k} a \wedge b & \text { iff } & a \oplus b=a \wedge b & \text { iff } \\ \langle a, b\rangle,\langle b, a\rangle \in \geq_{t} \circ \geq_{k} & \text { iff } & a \wedge b \geq_{t} a \oplus b & \text { iff } & a \wedge b=a \oplus b & \text { iff } \\ \langle a, b\rangle,\langle b, a\rangle \in \geq_{k} \circ \geq_{t} & \text { iff } & a \otimes b \geq_{k} a \vee b & \text { iff } & a \otimes b=a \vee b . & \end{array}$




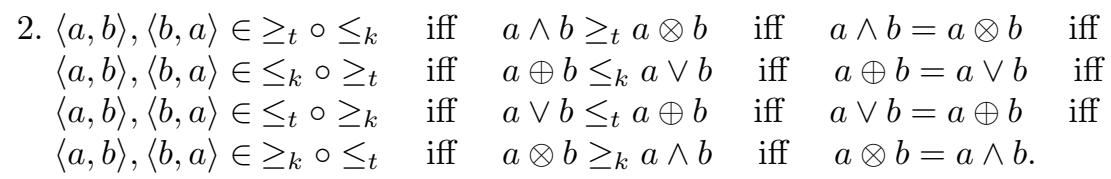

Proof. By the Duality Principle it is enough to prove the first statement. And using that we know that all four claims in the first column are equivalent indeed it is sufficient, again by the Duality Principle, to prove the part of this first statement saying that $\langle a, b\rangle,\langle b, a\rangle \in \leq_{t} \circ \leq_{k}$ iff $a \vee b \leq_{t} a \otimes b$ iff $a \vee b=a \otimes b$. And this is an easy consequence of Proposition 3.4 together with the fact that $a \otimes b \leq_{t} a \vee b$ by the interlacing conditions.

\section{DEFinition 3.7}

The equivalence relation $\sim_{1}$ is the one that relates two elements of a pre-bilattice B whenever any of the conditions in the first statement of Proposition 3.6 holds. Analogously, the equivalence relation $\sim_{2}$ is the one defined by the conditions in the second statement of the same proposition.

The following proposition contains the main results of this section: a direct decomposition of interlaced pre-bilattices, a representation as pre-bilattice products of two lattices and a characterization of the congruences. In the proof we shall use the fact that all varieties of (pre-)bilattices are congruence-distributive: this is immediate, since lattices are congruence-distributive [11, p. 87] and this property is preserved by expansions of the algebraic language.

Proposition 3.8

Let $\mathbf{B}=\langle B, \wedge, \vee, \otimes, \oplus\rangle$ be an interlaced pre-bilattice. Then

1. $\sim_{1}$ and $\sim_{2}$ are congruences of $\mathbf{B}$.

2. $\mathbf{B} / \sim_{1}$ and $\mathbf{B} / \sim_{2}$ are interlaced pre-bilattices.

3. In $\mathbf{B} / \sim_{1}$, the knowledge order coincides with the truth order. That is, $\langle B, \otimes, \oplus\rangle / \sim_{1}=$ $\langle B, \wedge, \vee\rangle / \sim_{1}$.

4. In $\mathbf{B} / \sim_{2}$, the knowledge order is the dual of the truth order. That is, $\langle B, \otimes, \oplus\rangle / \sim_{2}=$ $\langle B, \vee, \wedge\rangle / \sim_{2}$.

5. $\sim_{1}$ and $\sim_{2}$ is a pair of factor congruences of $\mathbf{B}$ (i.e., $\sim_{1} \cap \sim_{2}$ is the identity relation, and $\sim_{1} \circ \sim_{2}$ is the total relation $\nabla$ ).

6. $\mathbf{B}$ is isomorphic to the direct product $\mathbf{B} / \sim_{1} \times \mathbf{B} / \sim_{2}$.

7. $\mathbf{B}$ is isomorphic to the product pre-bilattice $\left(\langle B, \otimes, \oplus\rangle / \sim_{1}\right) \odot\left(\langle B, \otimes, \oplus\rangle / \sim_{2}\right)$.

8. $\langle\operatorname{Con}(\mathbf{B}), \subseteq\rangle$ is isomorphic to $\left\langle\operatorname{Con}\left(\langle B, \otimes, \oplus\rangle / \sim_{1}\right), \subseteq\right\rangle \times\left\langle\operatorname{Con}\left(\langle B, \otimes, \oplus\rangle / \sim_{2}\right), \subseteq\right\rangle$.

9. $\langle\operatorname{Con}(\mathbf{B}), \subseteq\rangle$ is isomorphic to $\left\langle\left[\sim_{1}, \nabla\right]_{\operatorname{Con}(\mathbf{B})}, \subseteq\right\rangle \times\left\langle\left[\sim_{2}, \nabla\right]_{\operatorname{Con}(\mathbf{B})}, \subseteq\right\rangle$, where $\left[\sim_{i}, \nabla\right]_{\operatorname{Con}(\mathbf{B})}=\left\{\theta \in \operatorname{Con}(\mathbf{B}): \sim_{i} \subseteq \theta\right\}$.

10. $\operatorname{Con}(\mathbf{B}) \cong \operatorname{Con}(\langle B, \wedge, \vee\rangle) \cong \operatorname{Con}(\langle B, \otimes, \oplus\rangle)$.

Proof. 1. This is obvious from the fact that each one of these two relations is determined by a quasi-order compatible with the operations.

2. This is trivial.

3. It is enough to realize that $a \wedge b \sim_{1} a \otimes b$ (or that $a \vee b \sim_{1} a \oplus b$ ). By the interlacing conditions we know that $a \wedge b \leq_{k} a \otimes b \leq_{t} a \wedge b$. Hence, $\langle a \wedge b, a \otimes b\rangle \in \leq_{t} \circ \leq_{k}$ and $\langle a \otimes b, a \wedge b\rangle \in \leq_{t} \circ \leq_{k}$. Thus, it holds that $a \wedge b \sim_{1} a \otimes b$. 
4. It suffices to check that $a \wedge b \sim_{2} a \oplus b$ (or that $a \vee b \sim_{2} a \otimes b$ ). By the interlacing conditions we know that $a \wedge b \leq_{t} a \oplus b \leq_{k} a \wedge b$. Hence, $\langle a \wedge b, a \oplus b\rangle \in \leq_{t} \circ \leq_{k}$ and $\langle a \oplus b, a \wedge b\rangle \in \leq_{t} \circ \leq_{k}$. Thus, it holds that $a \wedge b \sim_{2} a \oplus b$.

5. First of all, we consider the case of the identity relation. Let us assume that $a \sim_{1} b$ and $a \sim_{2} b$. Then, by Proposition 3.6 we know that $a \vee b=a \otimes b$ and $a \wedge b=a \otimes b$. Therefore, $a \vee b=a \wedge b$. Hence, $a=b$. Now it is time to prove that $\sim_{1} \circ \sim_{2}$ is the total relation. In order to do this it is enough to check that the element $c:=(a \wedge(a \oplus b)) \otimes(b \vee(a \oplus b))$ satisfies that $a \sim_{1} c$ and $b \sim_{2} c$. But using previous items in this proposition it is obvious that

$$
c=(a \wedge(a \oplus b)) \otimes(b \vee(a \oplus b)) \sim_{1}(a \wedge(a \vee b)) \wedge(b \vee(a \vee b)) \sim_{1} a \wedge(a \vee b) \sim_{1} a
$$

and that

$$
c=(a \wedge(a \oplus b)) \otimes(b \vee(a \oplus b)) \sim_{2}(a \wedge(a \wedge b)) \vee(b \vee(a \wedge b)) \sim_{2}(a \wedge b) \vee b \sim_{2} b
$$

6. By the previous item and [11, Theorem II.7.5].

7. This holds because $\mathbf{B} / \sim_{1} \times \mathbf{B} / \sim_{2}$ is exactly $\left(\langle B, \otimes, \oplus\rangle / \sim_{1}\right) \odot\left(\langle B, \otimes, \oplus\rangle / \sim_{2}\right)$. We point out that $\left(\langle B, \otimes, \oplus\rangle / \sim_{1}\right) \cong\left(\langle B, \wedge, \vee\rangle / \sim_{1}\right)$ and that $\left(\langle B, \otimes, \oplus\rangle / \sim_{2}\right) \cong$ $\left(\langle B, \vee, \wedge\rangle / \sim_{2}\right)$.

8. As we have observed, pre-bilattices are congruence-distributive; hence they also enjoy the Fraser-Horn-Hu property [25, Corollary 1]. This means that the lattice of congruences of a direct product is isomorphic to the direct product of the lattices of congruences of the factor algebras. We have then that $\operatorname{Con}(\mathbf{B}) \cong \operatorname{Con}\left(\mathbf{B} / \sim_{1} \times \mathbf{B} / \sim_{2}\right.$ )$\cong \operatorname{Con}\left(\mathbf{B} / \sim_{1}\right) \times \operatorname{Con}\left(\mathbf{B} / \sim_{2}\right)$. To finish the proof it is enough to observe that, as a consequence of (iii) and (iv), it holds that if $i \in\{1,2\}$ then $\operatorname{Con}\left(\mathbf{B} / \sim_{i}\right)=$ $\operatorname{Con}\left(\langle B, \otimes, \oplus\rangle / \sim_{i}\right)$.

9. The beginning of this proof is the same one than for the previous item. In order to finish it we use that, by [11, Theorem II.6.20], if $i \in\{1,2\}$ then $\operatorname{Con}\left(\mathbf{B} / \sim_{i}\right) \cong\left[\sim_{i}, \nabla\right]$.

10. By the Duality Principle it suffices to prove that $\operatorname{Con}(\mathbf{B}) \cong \operatorname{Con}(\langle B, \otimes, \oplus\rangle)$. Recall that, as observed in (viii), if $i \in\{1,2\}$ then $\operatorname{Con}\left(\mathbf{B} / \sim_{i}\right)=\operatorname{Con}\left(\langle B, \otimes, \oplus\rangle / \sim_{i}\right)$. Using the Fraser-Horn-Hu property, we have $\operatorname{Con}(\mathbf{B}) \cong \operatorname{Con}\left(\mathbf{B} / \sim_{1} \times \mathbf{B} / \sim_{2}\right) \cong$ $\operatorname{Con}\left(\mathbf{B} / \sim_{1}\right) \times \operatorname{Con}\left(\mathbf{B} / \sim_{2}\right) \cong \operatorname{Con}\left(\langle B, \otimes, \oplus\rangle / \sim_{1}\right) \times \operatorname{Con}\left(\langle B, \otimes, \oplus\rangle / \sim_{2}\right) \cong \operatorname{Con}(\langle B, \otimes, \oplus\rangle)$.

Item (7) of the previous proposition implies Theorem 3.2, which we aimed to prove. Let us also note that (10) could be strengthened, for the isomorphism involved is indeed the identity, so that we have $\operatorname{Con}(\mathbf{B})=\operatorname{Con}(\langle B, \wedge, \vee\rangle)=\operatorname{Con}(\langle B, \otimes, \oplus\rangle)$. An interesting consequence of $(10)$ is that any property that only depends on the lattice of congruences transfers straightforwardly from interlaced pre-bilattices to lattices and viceversa (this can be regarded as a generalization of the results of [29]). For instance, an interlaced pre-bilattice $\mathbf{B}=\langle B, \wedge, \vee, \otimes, \oplus\rangle$ is subdirectly irreducible if and only if any (hence both) of its lattice reducts $\langle B, \wedge, \vee\rangle$ and $\langle B, \otimes, \oplus\rangle$ is a subdirectly irreducible lattice; it is directly indecomposable as a pre-bilattice if and only if its lattice reducts are, and so on. Note also that, using the Fraser-Horn-Hu property [25] as in Proposition 3.8 (10), we get as a consequence that $\operatorname{Con}\left(\mathbf{L}_{\mathbf{1}} \odot \mathbf{L}_{\mathbf{2}}\right) \cong$ $\operatorname{Con}\left(\mathbf{L}_{1}\right) \times \operatorname{Con}\left(\mathbf{L}_{2}\right)$.

The following theorem provides another generalization of some results that are known for bounded interlaced pre-bilattices. Let $\varepsilon(\sqcap, \sqcup)$ be an equation in the lan- 
guage of lattices and, if $\{\circ, \bullet\}$ are connectives of the language of pre-bilattices, denote by $\varepsilon(\circ, \bullet)$ the result of substituting $\circ$ and $\bullet$ respectively for $\sqcap$ and $\sqcup$.

THEOREM 3.9

Let $\mathbf{B}$ be a pre-bilattice and let $\varepsilon(\sqcap, \sqcup)$ be an equation in the language of lattices. Then the following statements are equivalent:

1. $\mathbf{B}$ is an interlaced pre-bilattice such that $\mathbf{B}=\{\varepsilon(\wedge, \vee), \varepsilon(\vee, \wedge), \varepsilon(\otimes, \oplus), \varepsilon(\oplus, \otimes)\}$.

2. $\mathbf{B}$ is an interlaced pre-bilattice such that $\mathbf{B} \models\{\varepsilon(\wedge, \vee), \varepsilon(\vee, \wedge)\}$.

3. $\mathbf{B}$ is an interlaced pre-bilattice such that $\mathbf{B} \models\{\varepsilon(\otimes, \oplus), \varepsilon(\oplus, \otimes)\}$.

4. There are two lattices $\mathbf{L}_{\mathbf{1}}$ and $\mathbf{L}_{\mathbf{2}}$ such that $\mathbf{L}_{\mathbf{1}} \models\{\varepsilon(\sqcap, \sqcup), \varepsilon(\sqcup, \sqcap)\}, \mathbf{L}_{\mathbf{2}}=$ $\{\varepsilon(\sqcap, \sqcup), \varepsilon(\sqcup, \sqcap)\}$ and $\mathbf{B}$ is isomorphic to $\mathbf{L}_{\mathbf{1}} \odot \mathbf{L}_{\mathbf{2}}$.

Proof. By the duality it is enough to see that all conditions except the second one are equivalent.

$1 \Rightarrow 3$ : This is trivial.

$3 \Rightarrow 4$ : By the seventh item of Theorem 3.11.

$4 \Rightarrow 1$ : This follows from the fact that $\mathbf{L}_{\mathbf{1}} \odot \mathbf{L}_{\mathbf{2}} \cong \mathbf{L}_{\mathbf{1}}^{++} \times \mathbf{L}_{\mathbf{2}}^{-+}$.

\subsection{Representation theorem for interlaced bilattices}

In this subsection we state the corresponding representation theorem for interlaced bilattices, i.e. for the case where the language has been expanded with a negation operation. This fact has important consequences, in the first place that the two lattice factors obtained though the representation are isomorphic. Again, we point out that this result was already known for bounded interlaced bilattices.

First of all, let us note that the presence of negation affects the Duality Principle, for in this case we are only allowed to replace $\leq_{t}$ by $\geq_{t}$ and $\leq_{k}$ by $\geq_{k}$. It is no more allowed to switch $\leq_{t}$ and $\leq_{k}$ because negation has to be antimonotonic with respect to $\leq_{t}$ and monotonic with respect to $\leq_{k}$.

DEFINITION 3.10

Let $\mathbf{L}=\langle L, \sqcap, \sqcup\rangle$ be a lattice with associated order $\leq$. Then the product bilattice $\mathbf{L} \odot \mathbf{L}=\langle L \times L, \wedge, \vee, \otimes, \oplus, \neg\rangle$ is defined as in Definition 3.1 for the pre-bilattice reduct and the negation is given by

$$
\neg\left\langle a_{1}, a_{2}\right\rangle=\left\langle a_{2}, a_{1}\right\rangle .
$$

Negation is permuting the two components, hence it is obvious that this product construction is not a special case of direct product. It is easily checked that the algebra $\mathbf{L} \odot \mathbf{L}$ is always an interlaced bilattice. Now the representation theorem we want to prove is the following:

Theorem 3.11 (Representation)

Let $\mathbf{B}$ be a pre-bilattice. The following statements are equivalent.

1. $\mathbf{B}$ is an interlaced bilattice.

2. There is a lattice $\mathbf{L}$ such that $\mathbf{B}$ is isomorphic to $\mathbf{L} \odot \mathbf{L}$. 


\section{The logic of distributive bilattices}

The rest of this subsection is devoted to the proof of the above theorem. We will follow the same strategy of Section 3.1, that is, we shall consider the congruences $\sim_{1}$ and $\sim_{2}$ of the pre-bilattice reduct (but note that $\sim_{1}$ and $\sim_{2}$ are not compatible with the negation operator).

LEMMA 3.12

Let $\mathbf{B}$ be an interlaced bilattice. Then, for every $a, b \in B$ it holds that

1. $a \sim_{1} b$ iff $\neg a \sim_{2} \neg b$.

2. $a \sim_{2} b$ iff $\neg a \sim_{1} \neg b$.

Proof. This follows from Proposition 3.6 together with De Morgan laws.

Proposition 3.13

Let $\mathbf{B}=\langle B, \wedge, \vee, \otimes, \oplus, \neg\rangle$ be an interlaced bilattice. Then:

1. $\langle B, \otimes, \oplus\rangle / \sim_{1} \cong\langle B, \otimes, \oplus\rangle / \sim_{2}$.

2. $\mathbf{B}$ is isomorphic to the product bilattice $\left(\langle B, \otimes, \oplus\rangle / \sim_{1}\right) \odot\left(\langle B, \otimes, \oplus\rangle / \sim_{1}\right)$.

3. $\operatorname{Con}(\mathbf{B}) \cong \operatorname{Con}\left(\langle B, \otimes, \oplus\rangle / \sim_{1}\right)$.

4. $\operatorname{Con}(\mathbf{B}) \cong \operatorname{Con}(\langle B, \wedge, \vee, \neg\rangle) \cong \operatorname{Con}(\langle B, \otimes, \oplus, \neg\rangle)$.

Proof. 1. By Lemma 3.12 it is clear that the map defined by the assignment $[a]_{\sim_{1}} \longmapsto[\neg a]_{\sim_{2}}$ is an isomorphism.

2. By Proposition 3.8 we know that the map $a \longmapsto\left\langle[a]_{\sim_{1}},[a]_{\sim_{2}}\right\rangle$ is an isomorphism between $\langle B, \wedge, \vee, \otimes, \oplus\rangle$ and $\left(\langle B, \otimes, \oplus\rangle / \sim_{1}\right) \odot\left(\langle B, \otimes, \oplus\rangle / \sim_{2}\right)$ (this last product is taken as a pre-bilattice). Therefore, by the previous item we know that the map $a \longmapsto\left\langle[a]_{\sim_{1}},[\neg a]_{\sim_{1}}\right\rangle$ is an isomorphism between $\langle B, \wedge, \vee, \otimes, \oplus\rangle$ and $\left(\langle B, \otimes, \oplus\rangle / \sim_{1}\right.$ )$\odot\left(\langle B, \otimes, \oplus\rangle / \sim_{1}\right)$. Thus, it suffices to prove that this last map is also a homomorphism of the negation operator; and this is trivial.

3. By the previous item it is enough to prove that $\operatorname{Con}(\mathbf{L} \odot \mathbf{L}) \cong \operatorname{Con}(\mathbf{L})$. Let $\mathbf{L}$ be the lattice $\langle L, \sqcap, \sqcup\rangle$. We know from the pre-bilattice case (see a paragraph below Proposition 3.8) that $\operatorname{Con}(\langle L, \sqcap, \sqcup\rangle \odot\langle L, \sqcap, \sqcup\rangle) \cong \operatorname{Con}(\langle L, \sqcap, \sqcup\rangle) \times \operatorname{Con}(\langle L, \sqcap, \sqcup\rangle)$ under the assignment $\theta \longmapsto\left\langle\pi_{1}(\theta), \pi_{2}(\theta)\right\rangle$. Here $\pi_{i}$ refers to $i$ th-projection. If $\theta \in$ $\operatorname{Con}(\langle L, \sqcap, \sqcup\rangle \odot\langle L, \sqcap, \sqcup\rangle)$ is also a congruence with respect to the negation operator then $\pi_{1}(\theta)=\pi_{2}(\theta)$ because

$\left\langle a_{1}, a_{2}\right\rangle \in \pi_{1}(\theta) \quad$ iff $\quad$ there is some $b \in L$ such that $\left\langle a_{1}, b\right\rangle \theta\left\langle a_{2}, b\right\rangle \quad$ iff there is some $b \in L$ such that $\left\langle b, a_{1}\right\rangle \theta\left\langle b, a_{2}\right\rangle \quad$ iff $\quad\left\langle a_{1}, a_{2}\right\rangle \in \pi_{2}(\theta)$.

Therefore, the map $\theta \longmapsto \pi_{1}[\theta]$ is an isomorphism between $\operatorname{Con}(\langle L, \sqcap, \sqcup\rangle \odot\langle L, \sqcap, \sqcup\rangle)$ and $\operatorname{Con}(\langle L, \sqcap, \sqcup\rangle)$.

4. This is an easy consequence of Proposition 3.8 (10).

The second item of the above proposition implies Theorem 3.11. Note also that (4) could be strengthened, in the sense that we have

$$
\operatorname{Con}(\mathbf{B})=\operatorname{Con}(\langle B, \wedge, \vee, \neg\rangle)=\operatorname{Con}(\langle B, \otimes, \oplus, \neg\rangle)=\operatorname{Con}(\langle B, \wedge, \neg\rangle) .
$$

REMARK 3.14

In the case of interlaced bilattices it would be possible to give an alternative and straightforward proof of the representation theorem that still does not rely on the 
boundedness assumption. The role of negation in this simple proof is essential. The idea is to define a regular element as one that is a fixed point of negation, and to take the set $\operatorname{Reg}(\mathbf{B})=\{a \in B: a=\neg a\}$ of regular elements. It is easy to see that this set is closed under $\otimes, \oplus$, hence is the universe of a sublattice of the k-lattice of $\mathbf{B}$. Now, for every $a \in B$ we define

$$
\operatorname{reg}(a):=(a \vee(a \otimes \neg a)) \oplus \neg(a \vee(a \otimes \neg a)) .
$$

It is easy to check that for all $a, b \in B$,

- $a \in \operatorname{Reg}(\mathbf{B})$ iff $a=\operatorname{reg}(a)$ iff $a=\operatorname{reg}(b)$ for some $b \in B$,

- $a \sim_{1} \operatorname{reg}(a)$,

- $\langle a, b\rangle \in \leq_{t} \circ \leq_{k} \quad$ iff $\quad\langle\operatorname{reg}(a), \operatorname{reg}(b)\rangle \in \leq_{t} \circ \leq_{k} \quad$ iff $\quad \operatorname{reg}(a) \leq_{k} \operatorname{reg}(b)$,

- $a \sim_{1} b$ iff $\operatorname{reg}(a)=\operatorname{reg}(b)$,

- $\operatorname{reg}(a \otimes b)=\operatorname{reg}(\operatorname{reg}(a) \otimes \operatorname{reg}(b))=\operatorname{reg}(a) \otimes \operatorname{reg}(b)$,

- $\operatorname{reg}(a \oplus b)=\operatorname{reg}(\operatorname{reg}(a) \oplus \operatorname{reg}(b))=\operatorname{reg}(a) \oplus \operatorname{reg}(b)$.

To prove the second of these properties it is convenient to use the fact that

$$
\begin{gathered}
\operatorname{reg}(a) \sim_{1} a \vee(a \otimes \neg a) \\
a \vee \neg(a \vee(a \otimes \neg a))=a \vee(a \otimes \neg a) \vee(\neg a \wedge(a \otimes \neg a))= \\
a \vee \neg a) \sim_{1} a \oplus(a \otimes \neg a)=a .
\end{gathered}
$$

And now from these facts it follows that the map reg: $\langle B, \otimes, \oplus\rangle \longrightarrow\langle\operatorname{Reg}(\mathbf{B}), \otimes, \oplus\rangle$ is an epimorphism with kernel $\sim_{1}$. So $\langle B, \otimes, \oplus\rangle / \sim_{1}$ is isomorphic to $\langle\operatorname{Reg}(\mathbf{B}), \otimes, \oplus\rangle$. This suggests (cf. Proposition 3.13) that, as a different strategy to prove the representation theorem, we could have directly showed that $\mathbf{B} \cong\langle\operatorname{Reg}(\mathbf{B}), \otimes, \oplus\rangle \odot$ $\langle\operatorname{Reg}(\mathbf{B}), \otimes, \oplus\rangle$

To close this subsection, we state an analogue of Theorem 3.9 for bilattices.

THEOREM 3.15

Let $\mathbf{B}$ be a bilattice and let $\varepsilon(\sqcap, \sqcup)$ be an equation in the language of lattices. The following statements are equivalent.

1. $\mathbf{B}$ is an interlaced pre-bilattice such that $\mathbf{B}=\{\varepsilon(\wedge, \vee), \varepsilon(\vee, \wedge), \varepsilon(\otimes, \oplus), \varepsilon(\oplus, \otimes)\}$.

2. $\mathbf{B}$ is an interlaced pre-bilattice such that $\mathbf{B} \models\{\varepsilon(\wedge, \vee), \varepsilon(\vee, \wedge)\}$.

3. $\mathbf{B}$ is an interlaced pre-bilattice such that $\mathbf{B} \models\{\varepsilon(\otimes, \oplus), \varepsilon(\oplus, \otimes)\}$.

4. There are two lattices $\mathbf{L}_{\mathbf{1}}$ and $\mathbf{L}_{\mathbf{2}}$ such that $\mathbf{L}_{\mathbf{1}} \models\{\varepsilon(\sqcap, \sqcup), \varepsilon(\sqcup, \sqcap)\}, \mathbf{L}_{\mathbf{2}} \models$ $\{\varepsilon(\sqcap, \sqcup), \varepsilon(\sqcup, \sqcap)\}$ and $\mathbf{B}$ is isomorphic to $\mathbf{L}_{\mathbf{1}} \odot \mathbf{L}_{\mathbf{2}}$.

Proof. Similar to the proof of Theorem 3.9, except that now we use Proposition 3.13.

\subsection{The bifilter operator}

The bifilter closure system has not been extensively studied in the literature, not even in the bounded case ${ }^{11}$. In this Section we fill in this gap and establish some connections with what was done in Section 3.1.

\footnotetext{
${ }^{11}$ The only work we are aware of is [27].
} 


\section{The logic of distributive bilattices}

We remind the reader that bifilters of $\mathbf{B}$ are non-empty sets $F \subseteq B$ such that $F$ is a lattice filter both in $\leq_{t}$ and in $\leq_{k}$. Since the family $\{\emptyset\} \cup\{F \subseteq B: F$ is a bifilter of $\mathbf{B}\}$ is closed under arbitrary intersections ${ }^{12}$, we can associate a closure operator $\mathcal{F} \mathcal{F}$ with the previous closure system. It holds that $\mathcal{F} \mathcal{F}(\emptyset)=\emptyset$ and that if $X \neq \emptyset$ then $\mathcal{F} \mathcal{F}(X)$ is ${ }^{13}$ exactly the smallest bifilter expanding $X$. As usual, we write $\mathcal{F} \mathcal{F}(a)$ as an abbreviation for $\mathcal{F} \mathcal{F}(\{a\})$.

REMARK 3.16

Besides the bifilter closure operator one could consider the operators $\mathcal{I I}$ (ideal of both orders, also called biideal), $\mathcal{F} \mathcal{I}$ (filter of the t-order and ideal of the k-order) and $\mathcal{I F}$ (ideal of the t-order and filter of the k-order). But this is not necessary, because by Duality all these closure operators can be reduced to the bifilter operator as follows:

- $\mathcal{I} \mathcal{I}$ on a pre-bilattice $\mathbf{B}$ coincides with $\mathcal{F} \mathcal{F}$ over $\langle B, \vee, \wedge, \oplus, \otimes\rangle$

- $\mathcal{F} \mathcal{I}$ on a pre-bilattice $\mathbf{B}$ coincides with $\mathcal{F F}$ over $\langle B, \wedge, \vee, \oplus, \otimes\rangle$

- $\mathcal{I F}$ on a pre-bilattice $\mathbf{B}$ coincides with $\mathcal{F F}$ over $\langle B, \vee, \wedge, \otimes, \oplus\rangle$.

LEMMA 3.17

Let $\mathbf{B}$ be an interlaced (pre-)bilattice and let $X$ be a subset of $B$. Then:

$$
\begin{aligned}
\mathcal{F} \mathcal{F}(X) & =\left\{a \in B: \exists a_{1}, \ldots, a_{n} \in X \text { s.t. }\left\langle a_{1} \wedge \ldots \wedge a_{n}, a\right\rangle \in \leq_{t} \circ \leq_{k} \text { for some } n>0\right\} \\
& =\left\{a \in B: \exists a_{1}, \ldots, a_{n} \in X \text { s.t. }\left\langle a_{1} \otimes \ldots \otimes a_{n}, a\right\rangle \in \leq_{t} \circ \leq_{k} \text { for some } n>0\right\} .
\end{aligned}
$$

Proof. If $X$ is empty it is trivial, so suppose it is not and let

$$
F=\left\{a \in B: \exists a_{1}, \ldots, a_{n} \in X \text { s.t. }\left\langle a_{1} \wedge \ldots \wedge a_{n}, a\right\rangle \in \leq_{t} \circ \leq_{k} \text { for some } n>0\right\} .
$$

Note that, using Proposition 3.4 (1) and Corollary 3.5, it is not difficult to prove that $F$ is also equal to

$$
\left\{a \in B: \exists a_{1}, \ldots, a_{n} \in X \text { s.t. }\left\langle a_{1} \otimes \ldots \otimes a_{n}, a\right\rangle \in \leq_{t} \circ \leq_{k} \text { for some } n>0\right\} .
$$

In fact, if $\left\langle a_{1} \wedge \ldots \wedge a_{n}, a\right\rangle \in \leq_{t} \circ \leq_{k}$, then $\left\langle a_{1} \wedge \ldots \wedge a_{n}, a\right\rangle \in \leq_{k} \circ \leq_{t}$. By the interlacing conditions $a_{1} \otimes \ldots \otimes a_{n} \leq_{k} a_{1} \wedge \ldots \wedge a_{n}$, so $\left\langle a_{1} \otimes \ldots \otimes a_{n}, a\right\rangle \in \leq_{k} \circ \leq_{t}$, which is equivalent to $\left\langle a_{1} \otimes \ldots \otimes a_{n}, a\right\rangle \in \leq_{t} \circ \leq_{k}$. By symmetry we have that $\left\langle a_{1} \otimes \ldots \otimes a_{n}, a\right\rangle \in \leq_{t} \circ \leq_{k}$ implies $\left\langle a_{1} \wedge \ldots \wedge a_{n}, a\right\rangle \in \leq_{t} \circ \leq_{k}$, so the two conditions are equivalent. Now, to see that $F \subseteq \mathcal{F} \mathcal{F}(X)$, assume $a \in F$. This means that there are $a_{1}, \ldots, a_{n} \in X$ and $b \in B$ such that $a_{1} \wedge \ldots \wedge a_{n} \leq_{t} b \leq_{k} a$. Since $\mathcal{F} \mathcal{F}(X)$ is closed under $\wedge$, we have $a_{1} \wedge \ldots \wedge a_{n} \in \mathcal{F} \mathcal{F}(X)$, and since it is upward closed w.r.t. both lattice orderings, we have $b, a \in \mathcal{F F}(X)$ as well. Clearly $X \subseteq F$. Hence, in order to prove that $\mathcal{F} \mathcal{F}(X) \subseteq F$, it is sufficient to check that $F$ is a bifilter. That $F$ is closed under $\wedge$ follows immediately from the interlacing conditions; to show that it is closed under $\otimes$ we can use what we have proved in (3.1) above. Finally, that $F$ is upward closed w.r.t. both orders is also an immediate consequence of Corollary 3.5.

Thus, by the first item of Proposition 3.4 it is straightforward to prove that for every set $X \cup\{a, b\} \subseteq B$,

\footnotetext{
${ }^{12}$ If $\mathbf{B}$ is bounded, then it is not necessary to include the empty set in the previous family, but in order to be as general as possible we need to include it.

${ }^{13}$ This is a consequence of the fact that if $X \neq \emptyset$ then $\emptyset \neq \bigcap\{F \subset B: F$ is a bifilter of B such that $X \subseteq F\}$. This claim can be easily checked noting that if $a \in X$, then the set $\left\{x \in B: a \leq_{k} a \wedge x\right\}$, which we will consider considered below in greater detail, is a subset of $\bigcap\{F \subseteq B: F$ is a bifilter of $\mathbf{B}$ such that $X \subseteq F\}$.
} 
- $\mathcal{F F}(a)=\left\{x \in B: a \leq_{k} a \wedge x\right\}=\left\{x \in B: a \leq_{t} a \otimes x\right\}=\left\{x \in B: a \vee x \leq_{k} x\right\}=$ $\left\{x \in B: a \oplus x \leq_{t} x\right\}$,

- $a \sim_{1} b \quad$ iff $\quad \mathcal{F F}(a)=\mathcal{F F}(b)$,

- $\mathcal{F F}(a \vee b)=\mathcal{F} \mathcal{F}(a) \cap \mathcal{F F}(b)=\mathcal{F} \mathcal{F}(a \oplus b)$,

- $\mathcal{F} \mathcal{F}(a \wedge b)=\mathcal{F} \mathcal{F}(a) \vee \mathcal{F F}(b)=\mathcal{F} \mathcal{F}(a \otimes b)$,

- $\mathcal{F F}(X)=\left\{x \in B: a_{1} \wedge \ldots \wedge a_{n} \leq_{k} a_{1} \wedge \ldots \wedge a_{n} \wedge x\right.$ for some $\left.a_{1}, \ldots, a_{n} \in X\right\}=$ $\left\{x \in B: a_{1} \otimes \ldots \otimes a_{n} \leq_{t} a_{1} \otimes \ldots \otimes a_{n} \otimes x\right.$ for some $\left.a_{1}, \ldots, a_{n} \in X\right\}=$

$\left\{x \in B:\left(a_{1} \wedge \ldots \wedge a_{n}\right) \vee x \leq_{k} x\right.$ for some $\left.a_{1}, \ldots, a_{n} \in X\right\}=$

$\left\{x \in B:\left(a_{1} \otimes \ldots \otimes a_{n}\right) \oplus x \leq_{t} x\right.$ for some $\left.a_{1}, \ldots, a_{n} \in X\right\}$.

In particular we have seen that the relation $\sim_{1}$ defined in Section 3.1 is the one induced by the principal bifilters. Using the other three items of Proposition 3.4 we may obtain similar characterizations of $\mathcal{I} \mathcal{F}, \mathcal{F} \mathcal{I}$ and $\mathcal{I} \mathcal{I}$. Observe that for every $a, b \in B$,

$$
\begin{array}{lllll}
a \sim_{1} b & \text { iff } & \mathcal{F} \mathcal{F}(a)=\mathcal{F} \mathcal{F}(b) & \text { iff } & \mathcal{I I}(a)=\mathcal{I I}(b), \\
a \sim_{2} b & \text { iff } & \mathcal{F} \mathcal{I}(a)=\mathcal{F} \mathcal{I}(b) & \text { iff } & \mathcal{I} \mathcal{F}(a)=\mathcal{I} \mathcal{F}(b) .
\end{array}
$$

It is also worth pointing out that if $\mathbf{B}$ is a bilattice (so there is a negation operation), then $\mathcal{F F}(a)=\left\{x \in B: \operatorname{reg}(a) \leq_{k} \operatorname{reg}(x)\right\}$. Moreover ${ }^{14}$ we have $\mathcal{I F}(a)=\{x \in B$ : $\neg x \in \mathcal{F F}(\neg a)\}, \mathcal{F} \mathcal{I}(a)=\{x \in B: \neg a \in \mathcal{F} \mathcal{F}(\neg x)\}$ and $\mathcal{I} \mathcal{I}(a)=\{x \in B: a \in F F(x)\}$.

We end the section by studying the relationship between the bifilters of $\mathbf{L}_{\mathbf{1}} \odot \mathbf{L}_{\mathbf{2}}$ and the lattice filters of $\mathbf{L}_{\mathbf{1}}$ and $\mathbf{L}_{\mathbf{2}}$.

Proposition 3.18

Let $\mathbf{L}_{\mathbf{1}} \odot \mathbf{L}_{\mathbf{2}}$ be an interlaced (pre-)bilattice, where $\mathbf{L}_{\mathbf{1}}$ and $\mathbf{L}_{\mathbf{2}}$ are lattices. If $F$ is a nonempty subset of $L_{1} \times L_{2}$, then

1. $F$ is a bifilter of $\mathbf{L}_{\mathbf{1}} \odot \mathbf{L}_{\mathbf{2}}$ if and only if $\quad F=\bar{F} \times L_{2}$ for some lattice filter $\bar{F}$ of $\mathbf{L}_{1}$.

2. $F$ is a prime bifilter of $\mathbf{L}_{\mathbf{1}} \odot \mathbf{L}_{\mathbf{2}}$ if and only if $\quad F=\bar{F} \times L_{2}$ for some prime filter $\bar{F}$ of $\mathbf{L}_{\mathbf{1}}$.

Proof. 1. The leftwards implication is trivial. For the other direction let us assume that $F$ is a bifilter of $\mathbf{L}_{\mathbf{1}} \odot \mathbf{L}_{\mathbf{2}}$. Since $\pi_{1}[F]$ is obviously a lattice filter, it suffices to prove that $F=\pi_{1}[F] \times L_{2}$. The only non trivial inclusion to justify is that $\pi_{1}[F] \times L_{2} \subseteq F$. Hence, let us consider a pair $\langle a, b\rangle \in \pi_{1}[F] \times L_{2}$. Since $a \in \pi_{1}[F]$ we know that there is some $c \in L_{2}$ such that $\langle a, c\rangle \in F$. Now, using that

$$
\langle a, c\rangle \leq_{t}\left\langle a, b \sqcap_{2} c\right\rangle \leq_{k}\langle a, b\rangle
$$

together with the closure properties of a bifilter we get that $\langle a, b\rangle \in F$.

2. Again the leftwards direction is trivial, and hence we consider a prime bifilter $F$ of $\mathbf{L}_{\mathbf{1}} \odot \mathbf{L}_{\mathbf{2}}$ in order to prove the converse direction. By the previous item in this result we know that $F=\pi_{1}[F] \times L_{2}$. Thus, it suffices to prove that $\pi_{1}[F]$ is a prime lattice. Let us consider a pair of elements $a, b \in L_{1}$ such that $a \sqcup_{1} b \in \pi_{1}[F]$. Then for all $c \in L_{2}$, it holds that $\langle a, c\rangle \vee\langle b, c\rangle=\left\langle a \sqcup_{1} b, c\right\rangle \in \pi_{1}[F] \times L_{2}=F$. Using that

\footnotetext{
${ }^{14}$ Note that in the characterization of $\mathcal{I} \mathcal{I}(a)$ negation does not play any role (i.e., it also holds in pre-bilattices); it is written here for the sake of completeness.
} 


\section{The logic of distributive bilattices}

$L_{2}$ is non empty we get that $\langle a, c\rangle \vee\langle b, c\rangle \in F$ for some $c \in L_{2}$. Since $F$ is prime, this implies that either $\langle a, c\rangle \in F$ or $\langle b, c\rangle \in F$. Therefore we have that either $a \in \pi_{1}[F]$ or $b \in \pi_{1}[F]$.

An interesting consequence of the previous result is that the lattice of bifilters of an interlaced pre-bilattice $\mathbf{L}_{\mathbf{1}} \odot \mathbf{L}_{\mathbf{2}}$ is isomorphic to the lattice of filters of the first factor lattice $\mathbf{L}_{1}$ (note that the second factor $\mathbf{L}_{2}$ does not play any role). So, for instance, if $\mathbf{L}_{\mathbf{1}}$ is distributive (hence the lattice of its filters is distributive), then the lattice of bifilters of $\mathbf{L}_{\mathbf{1}} \odot \mathbf{L}_{\mathbf{2}}$ is also distributive. This result applies, in particular, to the class of distributive (pre-)bilattces that we study in the next section.

\subsection{The variety of distributive bilattices}

It is known that the variety of bounded distributive bilattices is generated by $\mathcal{F O U R}$. This was proved using the representation theorem for bounded bilattices together with the fact that the two-element lattice $\mathbf{2}$ generates the variety of bounded distributive lattices.

Having extended the representation theorem to the unbounded case, we can now easily obtain the corresponding result for unbounded bilattices.

\section{THEOREM 3.19}

- The variety DPreBiLat has two subdirectly irreducible algebras, i.e. $\mathbf{2}^{++}$and $\mathbf{2}^{-+}$, the two-element pre-bilattices whose direct product is the pre-bilattice reduct of $\mathcal{F O U R}$.

- The variety DPreBiLat is generated by its two-element members.

- The variety DBiLat has only one subdirectly irreducible algebra, i.e. $\mathcal{F O U R}$.

- The variety DBiLat is generated by $\mathcal{F O U R}$.

The previous results follow easily from Theorem 3.15, since distributive bilattices are, up to isomorphism, the product bilattices $\mathbf{L} \odot \mathbf{L}$ where $\mathbf{L}$ is a distributive lattice. Recall also that $\operatorname{Con}(\mathbf{L} \odot \mathbf{L}) \cong \operatorname{Con}(\mathbf{L})$. Hence, using the fact that the two-element lattice is the only subdirectly irreducible distributive lattice, we immediately obtain the following consequences.

Corollary 3.20

$\mathcal{F O U R}$ is the only subdirectly irreducible distributive bilattice.

Corollary 3.21

The variety DBiLat is generated by $\mathcal{F O U R}$.

In the remaining part of the section we shall prove some results on distributive prebilattices that can be regarded as analogues of well-known properties of distributive lattices.

Proposition 3.22

Let $\mathbf{B}$ be a distributive (pre-)bilattice. Then, for every set $X \cup\{a, b\} \subseteq B$, it holds that

$$
\mathcal{F} \mathcal{F}(X, a \vee b)=\mathcal{F} \mathcal{F}(X, a) \cap \mathcal{F F}(X, b)=\mathcal{F F}(X, a \oplus b) .
$$

Proof. By duality it is enough to prove that $\mathcal{F F}(X, a \vee b)=\mathcal{F} \mathcal{F}(X, a) \cap \mathcal{F F}(X, b)$. The inclusion $\mathcal{F} \mathcal{F}(X, a \vee b) \subseteq \mathcal{F} \mathcal{F}(X, a) \cap \mathcal{F} \mathcal{F}(X, b)$ is trivial, and next we prove the 
other inclusion. To this purpose let us assume that $c \in A$ is such that $c \in \mathcal{F} \mathcal{F}(X, a)$ and $c \in \mathcal{F} \mathcal{F}(X, b)$. Hence, there are some $a_{1}, \ldots, a_{n} \in X$ such that $c \in \mathcal{F} \mathcal{F}\left(a_{1} \wedge \ldots \wedge\right.$ $\left.a_{n} \wedge a\right)$ and $c \in \mathcal{F} \mathcal{F}\left(a_{1} \wedge \ldots \wedge a_{n} \wedge b\right)$. Thus, $\left\langle a_{1} \wedge \ldots \wedge a_{n} \wedge a, c\right\rangle \in \leq_{t} \circ \leq_{k}$ and $\left\langle a_{1} \wedge \ldots \wedge a_{n} \wedge b, c\right\rangle \in \leq_{t} \circ \leq_{k}$. Using that $\leq_{t} \circ \leq_{k}$ is a quasi order compatible with the operations it follows that $\left\langle a_{1} \wedge \ldots \wedge a_{n} \wedge(a \vee b), c\right\rangle=\left\langle\left(a_{1} \wedge \ldots \wedge a_{n} \wedge a\right) \vee\left(a_{1} \wedge\right.\right.$ $\left.\left.\ldots \wedge a_{n} \wedge b\right), c \vee c\right\rangle \in \leq_{t} \circ \leq_{k}$. Therefore, $c \in \mathcal{F} \mathcal{F}\left(a_{1} \wedge \ldots \wedge a_{n} \wedge(a \vee b)\right)$; and so $c \in \mathcal{F} \mathcal{F}(X, a \vee b)$.

Using Proposition 3.18, we may prove a Prime Bifilter Theorem for distributive (pre-)bilattices:

Proposition 3.23

Let $\mathbf{B}=\langle B, \wedge, \vee, \otimes, \oplus\rangle$ be a distributive pre-bilattice. Let $F$ be a non-empty proper bifilter and $I$ a biideal of $\mathbf{B}$ such that $F \cap I=\emptyset$. Then there exists a prime bifilter $P$ of $\mathbf{B}$ such that $F \subseteq P$ and $P \cap I=\emptyset$.

PROOF. By the previous results we may assume that $\mathbf{B} \cong \mathbf{L}_{\mathbf{1}} \odot \mathbf{L}_{\mathbf{2}}$ for some distributive lattices $\left\langle L_{1}, \sqcap_{1}, \sqcup_{2}\right\rangle$ and $\left\langle L_{2}, \sqcap_{2}, \sqcup_{2}\right\rangle$. Moreover, we know that $F=F_{1} \times L_{2}$ and $I=I_{1} \times L_{2}$, where $F_{1} \subseteq L_{1}$ is a lattice filter and $I_{1} \subseteq L_{1}$ is a lattice ideal. Since $L_{1}$ is distributive, by the Prime Filter Theorem we know that there is a prime filter $P_{1} \subseteq L_{1}$ such that $F_{1} \subseteq P_{1}$ and $P_{1} \cap I_{1}=\emptyset$. We claim that $P=P_{1} \times L_{2}$ is the desired prime bifilter. Clearly $F \subseteq P$, and by the previous results we know that $P$ is a prime bifilter. Moreover, if there were $\langle a, b\rangle \in P \cap I$, then we would have $a \in P_{1} \cap I_{1}$, against the hypothesis. Hence $P \cap I=\emptyset$ and we are done.

Using Proposition 3.23, it is easy to prove the following bifilter extension property:

Proposition 3.24

Let $\mathbf{B}=\langle B, \wedge, \vee, \otimes, \oplus\rangle$ be a distributive pre-bilattice. Let $F$ be a non-empty proper bifilter. Then there is a prime bifilter $P \in B$ such that $F \subseteq P \varsubsetneqq B$.

Proof. Assume $F \in B$ is a proper bifilter, i.e there is $a \in B$ s.t. $a \notin F$. Let $I=\mathcal{I} \mathcal{I}(a)$. Note that $F \cap I=\emptyset$. Indeed, if there were some $b \in F \cap I$, then by the former condition we would have $\mathcal{F F}(b) \subseteq F$, and by the latter we would have $a \oplus b \leq_{t} a$. Since $b \leq_{k} a \oplus b \leq_{t} a$, this implies that $a \in \mathcal{F} \mathcal{F}(b) \subseteq F$, against the assumption. Hence $F \cap I=\emptyset$. Now we can apply Proposition 3.23 and the result easily follows.

It is known that for lattices the Prime Filter Theorem is equivalent to distributivity. Hence, using Proposition 3.23, it is possible to prove that an interlaced pre-bilattice $\mathbf{L}_{\mathbf{1}} \odot \mathbf{L}_{\mathbf{2}}$ has the prime bifilter property if and only if $\mathbf{L}_{\mathbf{1}}$ is a distributive lattice. In fact, recalling Proposition 3.18, it is easy to see that if $\mathbf{L}_{\mathbf{1}} \odot \mathbf{L}_{\mathbf{2}}$ has the prime bifilter property, then $\mathbf{L}_{\mathbf{1}}$ has the prime filter property, hence is distributive; the converse is also easy. However, it is not true that if a pre-bilattice has the prime bifilter property then it is distributive: for a counterexample we just need to consider any pre-bilattice $\mathbf{L}_{\mathbf{1}} \odot \mathbf{L}_{\mathbf{2}}$ where $\mathbf{L}_{\mathbf{1}}$ is a distribuitve lattice while $\mathbf{L}_{\mathbf{2}}$ is not distributive. Note also that it is not true that given a pre-bilattice $\mathbf{B}$ and $a, b \in B$, if $a \neq b$ then there is a bifilter $F$ such that $a \in F$ and $b \notin F$. For instance, in a bounded pre-bilattice we have that $\top \in F$ iff $t \in F$ for every bifilter $F$. This is so because $t \leq_{k} \top$ and $\top \leq_{t} t$, so $t \in F$ implies $T \in F$ and conversely. However, it is possible to prove the following: 
26 The logic of distributive bilattices

Proposition 3.25

Let $\mathbf{B}=\langle B, \wedge, \vee, \otimes, \oplus\rangle$ be a distributive pre-bilattice and $a, b \in B$ s.t. $a \neq b$. Then either there is a prime bifilter $F \subset B$ such that $a \in F$ and $b \notin F$ or there is a prime t-ideal and k-filter $I \subset B$ such that $a \in I$ and $b \notin I$.

Proof. From the preceding results we know that if $\mathcal{F} \mathcal{F}(a)=\mathcal{F} \mathcal{F}(b)$ and $\mathcal{I I}(a)=$ $\mathcal{I I}(b)$, then $a=b$. By contraposition, we have that $a \neq b$ implies that either $\mathcal{F} \mathcal{F}(a) \neq$ $\mathcal{F F}(b)$ or $\mathcal{I} \mathcal{I}(a) \neq \mathcal{I} \mathcal{I}(b)$. Assume the first. By the Prime Bifilter Theorem we know that $\mathcal{F} \mathcal{F}(a)$ and $\mathcal{F} \mathcal{F}(b)$ are intersections of a family of prime bifilters, so there must be some prime bifilter $G$ in the family that is not in the other. So we have, for instance, $a \in G$ but $b \notin G$. Assuming the second, by the same reasoning we may conclude that there is a prime t-ideal and k-filter $H$ such that $a \in H$ but $b \notin H$.

Using the previous result, it is easy to obtain a representation theorem for distributive bilattices analogous to the well-known one for distributive lattices. Let us denote by $\operatorname{Pr} \mathcal{F} \mathcal{F}(\mathbf{B})$ the family of prime bifilters of a (pre-)bilattice $\mathbf{B}$ and by $\operatorname{Pr} \mathcal{I} \mathcal{F}(\mathbf{B})$ the family of prime t-ideal and k-filters of $\mathbf{B}$. Then we have the following:

COROllary 3.26

Every distributive (pre-)bilattice $\mathbf{B}$ is isomorphic to a sub(pre-)bilattice of the following (pre-)bilattice of sets:

$$
\langle P(\operatorname{Pr} \mathcal{F} \mathcal{F}(\mathbf{B})), \cap, \cup\rangle \odot\langle P(\operatorname{Pr} \mathcal{I} \mathcal{F}(\mathbf{B})), \cap, \cup\rangle
$$

through the map $\sigma$ defined, for all $a \in B$, as $\sigma(a)=\left\langle\sigma^{+}(a), \sigma^{-}(a)\right\rangle$, where

$$
\begin{aligned}
& \sigma^{+}(a)=\{F \subseteq B: F \text { is a prime bifilter and } a \in F\} ; \\
& \sigma^{-}(a)=\{I \subseteq B: I \text { is a prime t-ideal and k-filter and } a \in I\} .
\end{aligned}
$$

If $\mathbf{B}$ has a negation, then the map $\sigma^{-}$may also be defined as

$$
\sigma^{-}(a)=\{F \subseteq B: F \text { is a prime bifilter and } \neg a \in F\} .
$$




\section{AAL Study of $\mathcal{L B}$}

In this section we study the logic of logical bilattices from the point of view of Abstract Algebraic Logic. We will characterize the classes $\operatorname{A} \lg \mathcal{L B}$ and $\operatorname{Alg}^{*} \mathcal{L B}$ and compare them with the class of algebraic reducts of logical bilattices, which we denote by LoBiLat. Let us begin by classifying our logic according to the criteria of Abstract Algebraic Logic.

Proposition 4.1

The logic $\vDash_{\mathcal{L B}}$ is non-protoalgebraic and non-selfextensional.

Proof. Consider the bilattice $\mathcal{N} \mathcal{I N E}$ (Figure 1). The only proper and non-empty $\mathcal{L B}$-filters on $\mathcal{N} \mathcal{I N \mathcal { E }}$ are $F_{1}=\{e, \top, \mathrm{t}\} \subseteq\{b, c, d, e, \top, \mathrm{t}\}=F_{2}$. It is easy to check that $\langle\mathrm{t}, e\rangle \in \Omega\left\langle\mathcal{N} \mathcal{I} \mathcal{N} \mathcal{E}, F_{1}\right\rangle$ but $\langle\mathrm{t}, e\rangle \notin \Omega\left\langle\mathcal{N} \mathcal{I} \mathcal{N} \mathcal{E}, F_{2}\right\rangle$. That is, the Leibniz operator is not monotone on $\mathcal{L B}$-filters. As shown in [9], this implies that the logic $\vDash_{\mathcal{L B}}$ is not protoalgebraic. To be selfextensional means that the interderivability relation $==_{\mathcal{L B}}$ is a congruence of the formula algebra $\mathbf{F m}$. Now for any $p, q \in F m$ we have $p \oplus q=\|_{\mathcal{L B}} p \vee q$, but we can easily check that do not have $\neg(p \oplus q)=\|_{\mathcal{L B}} \neg(p \vee q)$. For instance in $\mathcal{F} \mathcal{O U} \mathcal{R}$ we have $\neg(t \oplus \top)=\top \in\{t, \top\}$ but $\neg(t \vee \top)=f \notin\{t, \top\}$.

\subsection{The algebraic counterpart of $\mathcal{L B}$}

The fact that $\mathcal{L B}$ is not selfextensional constitutes one of the main difficulties for an Abstract Algebraic Logic approach to $\mathcal{L B}$. As we have seen, this is due to the negation operator, and it is possible to see that this exception to selfextensionality is essentially the only one. We need the following lemmas.

LEMMA 4.2

Let $\varphi, \psi \in F m$ be two formulas. The following statements are equivalent:

1. $\mathcal{F} \mathcal{O U R} \vDash \varphi \wedge(\varphi \otimes \psi) \approx \varphi$,

2. $\varphi \vdash_{H} \psi$.

Proof. $1 \Rightarrow 2$. Let $h: \mathbf{F m} \rightarrow \mathcal{F O U R}$ be a homomorphism. If $h(\varphi)=\mathrm{t}$, then $\mathrm{t} \otimes h(\psi)=\mathrm{t}$, i.e. $h(\psi) \geq_{k} \mathrm{t}$, therefore $h(\psi) \in\{\top$, $\mathrm{t}\}$. If $h(\varphi)=\top$, then $\top \wedge h(\psi)=\top$, i.e. $h(\psi) \geq_{t} \top$, hence $h(\psi) \in\{\top$, t $\}$.

$2 \Rightarrow 1$. Let $h: \mathbf{F m} \rightarrow \mathcal{F O U R}$ be a homomorphism and assume that $\varphi \vdash_{H} \psi$.

If $h(\varphi)=\mathrm{t}$, then $h(\psi) \in\{\top$, t $\}$. So we must have $h(\varphi) \wedge(h(\varphi) \otimes h(\psi))=\mathrm{t} \otimes h(\psi)=\mathrm{t}$, i.e. $h(\psi) \geq_{k} \mathrm{t}$ and this is obvious.

If $h(\varphi)=\top$, then $h(\psi) \in\{\top$, t $\}$. So we must have $h(\varphi) \wedge(h(\varphi) \otimes h(\psi))=\top \wedge h(\psi)=$ $\top$, i.e. $h(\psi) \geq_{t} \top$ which is again obvious.

If $h(\varphi)=\perp$, then $h(\varphi) \wedge(h(\varphi) \otimes h(\psi))=\perp \wedge \perp=\perp=h(\varphi)$. Finally, the case where $h(\varphi)=\mathrm{f}$ is immediate.

As an immediate consequence of the preceding result, we have the following:

LEMMA 4.3

Let $\varphi, \psi \in F m$ be two formulas. The following statements are equivalent:

1. $\mathcal{F O U R} \vDash \varphi \approx \psi$,

2. $\varphi \vdash_{H} \psi$ and $\neg \varphi \dashv \vdash_{H} \neg \psi$. 


\section{The logic of distributive bilattices}

Proof. The only nontrivial implication is $2 \Rightarrow 1$. By Lemma 4.2 , we have that in $\mathcal{F O U R}$ the following equations hold:

(1) $\varphi \wedge(\varphi \otimes \psi) \approx \varphi$,

(2) $\psi \wedge(\varphi \otimes \psi) \approx \psi$

(3) $\neg \psi \wedge(\neg \varphi \otimes \neg \psi) \approx \neg \psi$,

(4) $\neg \varphi \wedge(\neg \varphi \otimes \neg \psi) \approx \neg \varphi$.

Negating both sides of (3) and using De Morgan laws, we obtain $\neg \neg \psi \approx \psi \approx \neg(\neg \psi \wedge$ $(\neg \varphi \otimes \neg \psi)) \approx \neg \neg \psi \vee \neg(\neg \varphi \otimes \neg \psi) \approx \psi \vee(\neg \neg \varphi \otimes \neg \neg \psi) \approx \psi \vee(\varphi \otimes \psi)$. From this and (2) it follows $\psi \approx \varphi \otimes \psi$. A similar reasoning shows that (1) and (4) imply $\varphi \approx \varphi \otimes \psi$. Hence $\varphi \approx \psi$.

The preceding result enables us to characterize the Tarski congruence associated with $\mathcal{L B}$ as the one defined by the equations valid in $\mathcal{F O U R}$ :

\section{THEOREM 4.4}

The Tarski congruence associated with $\mathcal{L B}$ is $\widetilde{\Omega}(\mathcal{L B})=\{\langle\varphi, \psi\rangle: \mathcal{F O U R} \vDash \varphi \approx \psi\}$.

Proof. Obviously the relation $\{\langle\varphi, \psi\rangle: \mathcal{F O U \mathcal { R }} \vDash \varphi \approx \psi\}$ is a congruence, and by Lemma 4.3 is is also clear that it is the maximal congruence below the Frege relation.

Recalling [22, Propositions 1.23 and 2.26], we can conclude that both $\operatorname{Alg}^{*} \mathcal{L B}$ and $\operatorname{Alg} \mathcal{L B}$ generate the same variety as $\mathcal{F O U} \mathcal{R}$ (i.e. the variety DBiLat of distributive bilattices). Moreover, we have the following:

THEOREM 4.5

$\operatorname{Alg} \mathcal{L B}$ is the variety generated by $\mathcal{F} \mathcal{O U} \mathcal{R}$, i.e. $\operatorname{Alg} \mathcal{L B}=$ DBiLat.

Proof. Clearly $\mathcal{F} \mathcal{O U} \mathcal{R} \in \mathbf{A} \lg * \mathcal{L B} \subseteq \operatorname{Alg} \mathcal{L B}$. By [22, Theorem 2.23] we also have $\mathcal{F O U R} \in \operatorname{Alg} \mathcal{L B}=P_{s}\left(\mathbf{A l g}^{*} \mathcal{L B}\right) \subseteq V(\mathcal{F O U R})$. Recall that $V(\mathcal{F O U R})=$ DBiLat is congruence-distributive. Hence we may apply Jónsson's Lemma [11, Corollary IV.6.10] to conclude that the subdirectly irreducible members of $V(\mathcal{F O U R})$ belong to $H S(\mathcal{F O U R})$, and clearly the only algebras in $H S(\mathcal{F O U R})$ are the trivial one and $\mathcal{F O U R}$ itself. Then we may conclude that $V(\mathcal{F O U R})=P_{s}(\mathcal{F O U R}) \subseteq P_{s}\left(\mathbf{A l g}^{*} \mathcal{L B}\right)$. Hence $P_{s}\left(\mathbf{A l g}^{*} \mathcal{L B}\right)=\operatorname{Alg} \mathcal{L B}=V(\mathcal{F O U R})$.

As an immediate corollary, we have that LoBiLat $\not \subset \operatorname{Alg} \mathcal{L B}$. This is true because $\langle\mathcal{D E F} \mathcal{A} \mathcal{U} \mathcal{L} \mathcal{T},\{\top, \mathrm{t}\}\rangle$ is a logical bilattice, but $\mathcal{D E \mathcal { F }} \mathcal{A U} \mathcal{L} \mathcal{T} \notin \operatorname{Alg} \mathcal{L B}$ since this bilattice is not distributive. We can also verify that $\mathcal{N} \mathcal{I} \mathcal{N E} \in \operatorname{Alg} \mathcal{L B}$ since it is distributive. Taking into account the previous results, this last claim follows from the fact that $\mathcal{N} \mathcal{I N} \mathcal{E} \cong \mathbf{3} \odot \mathbf{3}$, where $\mathbf{3}$ denotes the three-element lattice, which is a distributive lattice.

In order to describe the class of generalized models of $\mathcal{L B}$ (i.e. generalized matrices that are models of $\mathcal{L B}$; see [37]), we shall use the following characterization of $\mathcal{L B}$ filters:

Proposition 4.6

Let $\mathbf{B}$ be a distributive bilattice and $F \subseteq B$. Then $F$ is an $\mathcal{L B}$-filter if and only if $F$ is a bifilter of $\mathbf{B}$ or $F=\emptyset$. 
Proof. For $F$ empty the proof is trivial, so assume it is not. By rules (R3), (R4), (R3') and (R4') of our Hilbert calculus $\vdash_{H}$, it is obvious that any $\mathcal{L B}$-filter on $\mathbf{B}$ is a bifilter. It is also easy to see that, in a distributive bilattice, any bifilter is closed w.r.t. all rules of our Hilbert calculus. To see that it is closed under (R18) and (R19), recall that any interlaced (hence, any distributive) bilattice satisfies that $a \vee b \leq_{k} a \oplus b$ and $a \oplus b \leq_{t} a \vee b$ for all $a, b \in B$. Therefore, since any bifilter $F$ is upward closed w.r.t. both lattice orders, we have that $a \vee b \in F$ iff $a \oplus b \in F$.

Combining the result of the previous proposition with that of Theorem 4.5, we immediately obtain the following:

Corollary 4.7

If a generalized matrix $\langle\mathbf{A}, \mathcal{C}\rangle$ is a reduced generalized model of $\mathcal{L B}$, then $\mathbf{A}$ is a distributive bilattice and any non-empty $F \in \mathcal{C}$ is a bifilter.

One may wonder if the result of Corollary 4.7 could be strengthened, proving that if a generalized matrix $\langle\mathbf{A}, \mathcal{C}\rangle$ is a reduced generalized model of $\mathcal{L B}$, then $\mathbf{A}$ is a distributive bilattice and $\mathcal{C}$ is the family of all bifilters of $\mathbf{A}$ (possibly including the empty set). This is not the case, as we shall see later (Example 4.14).

Having individuated a class which, according to the general theory of [22], may be regarded as the algebraic counterpart of the logic $\mathcal{L B}$, we may wonder if this class could also be the algebraic counterpart of some other logic. Thanks to the general results of [10], in some cases one may be able to prove that a certain class of algebras cannot be the equivalent algebraic semantics of any algebraizable logic (such a result has been obtained, for instance, for the varieties of distributive bilattices and of De Morgan lattices: see $[24,20])$. This, however, is not the case with distributive bilattices, for it is possible to define a logic which is algebraizable w.r.t. the class DBiLat. Consider the following:

\section{EXAMPLE 4.8}

Let $\mathcal{R} e g=\left\langle\mathbf{F m}, \vdash_{\mathcal{R} e g}\right\rangle$ be the logic defined, for all $\Gamma \cup\{\varphi\} \subseteq F m$, as follows: $\Gamma \vdash_{\mathcal{R} e g} \varphi$ iff $\tau(\Gamma) \vDash_{\text {DBiLat }} \tau(\varphi)$, where $\tau$ is a translation from formulas into equations defined as $\tau(\varphi)=\{\varphi \approx \neg \varphi\}$ for all $\varphi \in F m$. By definition, the least $\mathcal{R} e g$-filter on any distributive bilattice coincides with the set of regular elements (whence the name chosen for the logic). It also follows from the definition that $\mathcal{R} e g$ satisfies one of the two conditions for being algebraizable w.r.t. the variety DBiLat, hence it will be sufficient to show that it satisfies the other one as well, namely the existence of a translation $\rho$ from equations into formulas s.t. $\varphi \approx \psi==_{\text {DBiLat }} \tau(\rho(\varphi \approx \psi))$. Defining $\rho(\varphi \approx \psi)=\{\neg \varphi \otimes \psi,(\varphi \oplus \neg \varphi) \wedge(\psi \oplus \neg \psi)\}$, the condition is satisfied. In fact, we have to prove that

$\varphi \approx \psi=\|_{\text {DBiLat }}\{\neg \varphi \otimes \psi \approx \neg(\neg \varphi \otimes \psi),(\varphi \oplus \neg \varphi) \wedge(\psi \oplus \neg \psi) \approx \neg((\varphi \oplus \neg \varphi) \wedge(\psi \oplus \neg \psi))\}$.

One direction is immediate; for the other, note that $\neg \varphi \otimes \psi \approx \neg(\neg \varphi \otimes \psi)$ is equivalent to $\neg \varphi \otimes \psi \approx \varphi \otimes \neg \psi$ and $(\varphi \oplus \neg \varphi) \wedge(\psi \oplus \neg \psi) \approx \neg((\varphi \oplus \neg \varphi) \wedge(\psi \oplus \neg \psi))$ is equivalent to $\varphi \oplus \neg \varphi \approx \psi \oplus \neg \psi$. Now let $\mathbf{B} \in$ DBiLat and $a, b \in \mathbf{B}$ such that $\neg a \otimes b=a \otimes \neg b$ and $a \oplus \neg a \approx b \oplus \neg b$. Using the absorption and the distributive laws, we obtain $a=a \otimes(a \oplus \neg a)=a \otimes(b \oplus \neg b)=(a \otimes b) \oplus(a \otimes \neg b)=(a \otimes b) \oplus(\neg a \otimes b)=$ $b \otimes(a \oplus \neg a)=b \otimes(b \oplus \neg b)=b$. 
30 The logic of distributive bilattices

\subsection{Reduced models of $\mathcal{L B}$}

In order to characterize the class of matrix models of $\mathcal{L B}$, we will now turn to the study of the Leibniz congruence of $\mathcal{L B}$.

PROPOSITION 4.9

Let $\langle\mathbf{A}, F\rangle$ be a model the logic $\mathcal{L B}$. Then, for all $a, b \in A$, the following are equivalent:

1. $\langle a, b\rangle \in \boldsymbol{\Omega}_{\mathbf{A}}(F)$,

2. $\{c \in A: a \vee c \in F\}=\{c \in A: b \vee c \in F\}$ and $\{c \in A: \neg a \vee c \in F\}=\{c \in A$ : $\neg b \vee c \in F\}$.

3. $\{c \in A: a \oplus c \in F\}=\{c \in A: b \oplus c \in F\}$ and $\{c \in A: \neg a \oplus c \in F\}=\{c \in A:$ $\neg b \oplus c \in F\}$.

Proof. $1 \Rightarrow 2$. It is easy to see that any congruence $\theta$ compatible with $F$ must satisfy 2. For instance, if $\langle a, b\rangle \in \theta$, then, for any $c \in A$, we have $\langle a \vee c, b \vee c\rangle \in \theta$ as well. Hence we have that $a \vee c \in F$ if and only if $b \vee c \in F$. A similar argument shows also that 1 implies 3 .

$2 \Rightarrow 1$. Let $\theta$ be the relation defined by the conditions of 2 , that is, for all $a, b \in A$, we set $\langle a, b\rangle \in \theta$ if and only if $\{c \in A: a \vee c \in F\}=\{c \in A: b \vee c \in F\}$ and $\{c \in A: \neg a \vee c \in F\}=\{c \in A: \neg b \vee c \in F\}$. Clearly, to prove that $\theta \subseteq \boldsymbol{\Omega}_{\mathbf{A}}(F)$, it is sufficient to check that $\theta$ is a congruence compatible with $F$. Taking into account the fact that $F$ is an $\mathcal{L B}$-filter, it is not difficult to see that $\theta$ is a congruence. We need to prove, for instance, that $\left\langle a_{1}, b_{1}\right\rangle,\left\langle a_{2}, b_{2}\right\rangle \in \theta$ implies $\left\langle a_{1} \wedge a_{2}, b_{1} \wedge b_{2}\right\rangle \in \theta$. For this, assume $\left(a_{1} \wedge a_{2}\right) \vee c \in F$ for some $c \in A$. This implies

$$
\begin{aligned}
& c \vee\left(a_{1} \wedge a_{2}\right) \in F \quad \text { by (R5) } \\
& \left(c \vee a_{1}\right) \wedge\left(c \vee a_{2}\right) \in F \quad \text { by (R8) } \\
& \left(c \vee a_{1}\right),\left(c \vee a_{2}\right) \in F \quad \text { by (R1) and (R2) } \\
& \left(a_{1} \vee c\right),\left(a_{2} \vee c\right) \in F \quad \text { by (R5) } \\
& \left(b_{1} \vee c\right),\left(b_{2} \vee c\right) \in F \quad \text { by definition of } \theta \\
& \left(c \vee b_{1}\right),\left(c \vee b_{2}\right) \in F \quad \text { by (R5) } \\
& \left(c \vee b_{1}\right) \wedge\left(c \vee b_{2}\right) \in F \quad \text { by (R3) } \\
& c \vee\left(b_{1} \wedge b_{2}\right) \in F \quad \text { by (R9) } \\
& \left(b_{1} \wedge b_{2}\right) \vee c \in F \quad \text { by (R5). }
\end{aligned}
$$

Hence the first condition of 2 is satisfied. A similar argument allows to prove the second one as well, so that we may conclude that $\left\langle a_{1} \wedge a_{2}, b_{1} \wedge b_{2}\right\rangle \in \theta$. To see that $\theta$ is compatible with $F$, assume $\langle a, b\rangle \in \theta$ and $a \in F$. We have:

$$
\begin{aligned}
a \vee b & \in F & \text { by (R4) } \\
b \vee b & \in F & \text { by definition of } \theta \\
b & \in F & \text { by (R6). }
\end{aligned}
$$

$2 \Leftrightarrow 3$. Almost immediate, since by (R18) and (R19) we have that $a \vee b \in F$ iff $a \oplus b \in F$ for any $a, b \in A$ and any $\mathcal{L B}$-filter $F$. 
As a consequence of Proposition 4.9, we obtain the following characterization of the reduced matrix models of $\mathcal{L B}$ :

Theorem 4.10

Let $\mathbf{A}$ be a non-trivial algebra. The following conditions are equivalent:

1. $\langle\mathbf{A}, F\rangle$ is a reduced matrix for $\mathcal{L B}$,

2. A $\in$ DBiLat and $F$ is a bifilter s.t., for all $a, b \in A$, if $a<_{t} b$, then there is $c \in A$ s.t. either $a \vee c \notin F$ and $b \vee c \in F$ or $\neg a \vee c \in F$ and $\neg b \vee c \notin F$,

3. A $\in$ DBiLat and $F$ is a bifilter s.t., for all $a, b \in A$, if $a<_{k} b$, then there is $c \in A$ s.t. either $a \vee c \notin F$ and $b \vee c \in F$ or $\neg a \vee c \notin F$ and $\neg b \vee c \in F$.

Proof. $1 \Rightarrow 2$. Assume $\langle\mathbf{A}, F\rangle$ is a reduced matrix for $\mathcal{L B}$. That $\mathbf{A} \in$ DBiLat follows from Theorem 4.5, while Proposition 4.6 implies that $F$ is a bifilter (the assumption that $\mathbf{A}$ is not trivial guarantees that $F \neq \emptyset$ ). Notice that $a<_{t} b$ implies that $b \in \mathcal{F} \mathcal{F}(a)$ and $\neg a \in \mathcal{F} \mathcal{F}(\neg b)$; obviously it also implies that $\langle a, b\rangle \notin \boldsymbol{\Omega}_{\mathbf{A}}(F)$. By Proposition 4.9, this means that either $\{c \in A: a \vee c \in F\} \neq\{c \in A: b \vee c \in F\}$ or $\{c \in A: \neg a \vee c \in F\} \neq\{c \in A: \neg b \vee c \in F\}$. If the first is the case, then, for some $c \in A$, either $a \vee c \notin F$ and $b \vee c \in F$ or $a \vee c \in F$ and $b \vee c \notin F$. The latter hypothesis is impossible, for $b \in \mathcal{F F}(a)$ implies $\mathcal{F} \mathcal{F}(b \vee c)=\mathcal{F F}(b) \cap \mathcal{F F}(c) \subseteq$ $\mathcal{F} \mathcal{F}(a) \cap \mathcal{F} \mathcal{F}(c)=\mathcal{F} \mathcal{F}(a \vee c)$. So if $a \vee c \in F$, then $b \vee c \in F$ for any bifilter $F$. Hence the former hypothesis must be true. A similar argument can be applied to the case of $\{c \in A: \neg a \vee c \in F\} \neq\{c \in A: \neg b \vee c \in F\}$. Recalling that $a<_{k} b$ implies $b \in \mathcal{F F} \mathcal{F}(a)$ and $\neg b \in \mathcal{F F}(\neg a)$, it is easy to apply the same reasoning in order to show also that $1 \Rightarrow 3$.

$2 \Rightarrow 1$. Assume that $\mathbf{A} \in$ DBiLat and $F$ is a bifilter satisfying (ii). Assume also $a \neq b$. Then $a \wedge b<_{t} a \vee b$, hence we may apply the assumption and Proposition 4.9 to conclude that $\langle a \wedge b, a \vee b\rangle \notin \Omega_{\mathbf{A}}(F)$. Since we are in a lattice, this implies $\langle a, b\rangle \notin \boldsymbol{\Omega}_{\mathbf{A}}(F)$. Hence $\boldsymbol{\Omega}_{\mathbf{A}}(F)=I d_{\mathbf{A}}$. A similar reasoning shows that $3 \Rightarrow 1$.

Notice that, using the characterization given by item 3 instead of 2 of Proposition 4.9 , we could equivalently formulate conditions 2 and 3 of Theorem 4.10 using $\oplus$ instead of $\vee$, thus obtaining the following:

\section{Corollary 4.11}

Let $\mathbf{A}$ be a non-trivial algebra. The following conditions are equivalent:

1. $\langle\mathbf{A}, F\rangle$ is a reduced matrix for $\mathcal{L B}$,

2. A $\in$ DBiLat and $F$ is a bifilter s.t., for all $a, b \in A$, if $a<_{t} b$, then there is $c \in A$ s.t. either $a \oplus c \notin F$ and $b \oplus c \in F$ or $\neg a \oplus c \in F$ and $\neg b \oplus c \notin F$,

3. A $\in$ DBiLat and $F$ is a bifilter s.t., for all $a, b \in A$, if $a<_{k} b$, then there is $c \in A$ s.t. either $a \oplus c \notin F$ and $b \oplus c \in F$ or $\neg a \oplus c \notin F$ and $\neg b \oplus c \in F$.

We know that all algebras in $\operatorname{Alg}^{*} \mathcal{L B}$ are distributive bilattices, hence isomorphic to a product bilattice of the form $\mathbf{L} \odot \mathbf{L}$. The following lemma enables us to determine which requirements $\mathbf{L}$ must satisfy in order to have $\mathbf{L} \odot \mathbf{L} \in \mathbf{A} \mathbf{l g}^{*} \mathcal{L B}$.

LEMMA 4.12

Let $\mathbf{L} \odot \mathbf{L}=\langle L \times L, \wedge, \vee, \otimes, \oplus, \neg\rangle$ be an interlaced bilattice, where $\mathbf{L}=\langle L, \sqcap, \sqcup\rangle$ is a lattice. Let $F \subseteq L$ be a lattice filter of $\mathbf{L}$ and $\theta \in \operatorname{Con}(\mathbf{L} \odot \mathbf{L})$. Then $\theta$ is compatible 
with $F \times L$ if and only if $\pi(\theta)$ is compatible with $F$, where

$$
\pi(\theta)=\{\langle a, b\rangle \in L \times L: \exists c \in L \text { s.t. }\langle\langle a, c\rangle,\langle b, c\rangle\rangle \in \theta\} .
$$

As a consequence, we have that $\boldsymbol{\Omega}_{\mathbf{L} \odot \mathbf{L}}(F \times L)=I d_{L \times L}$ if and only if $\boldsymbol{\Omega}_{\mathbf{L}}(F)=I d_{L}$.

Proof. Note first that, by Proposition 3.18, we know that $F \times L$ is a bifilter of $\mathbf{L} \odot \mathbf{L}$. Now, for the first claim, suppose $\theta \in \operatorname{Con}(\mathbf{L} \odot \mathbf{L})$ is compatible with $F \times L, a \in F$ and $\langle a, b\rangle \in \pi(\theta)$. By the definition of $\pi(\theta)$ we have that there is $c \in L$ such that $\langle\langle a, c\rangle,\langle b, c\rangle\rangle \in \theta$, so by the compatibility of $\theta$ we obtain $\langle b, c\rangle \in F \times L$. Hence $b \in F$.

Conversely, suppose $\pi(\theta)$ is compatible with $F \subseteq L,\left\langle a_{1}, a_{2}\right\rangle \in F \times L$ and $\left\langle\left\langle a_{1}, a_{2}\right\rangle\right.$, $\left.\left\langle b_{1}, b_{2}\right\rangle\right\rangle \in \theta$. Note that $\left\langle\left\langle a_{1}, a_{2} \sqcap b_{2}\right\rangle,\left\langle b_{1}, a_{2} \sqcap b_{2}\right\rangle\right\rangle \in \theta$, because $\theta$ is a congruence and

$$
\left\langle a_{1}, a_{2}\right\rangle \otimes\left\langle a_{1} \sqcup b_{1}, a_{2} \sqcap b_{2}\right\rangle=\left\langle a_{1} \sqcap\left(a_{1} \sqcup b_{1}\right), a_{2} \sqcap\left(a_{2} \sqcap b_{2}\right)\right\rangle=\left\langle a_{1}, a_{2} \sqcap b_{2}\right\rangle .
$$

Similarly we obtain $\left\langle b_{1}, b_{2}\right\rangle \otimes\left\langle a_{1} \sqcup b_{1}, a_{2} \sqcap b_{2}\right\rangle=\left\langle b_{1}, a_{2} \sqcap b_{2}\right\rangle$. By definition, this means that $\left\langle a_{1}, b_{1}\right\rangle \in \pi(\theta)$. So, by the compatibility of $\pi(\theta)$, we obtain $b_{1} \in F$, i.e. $\left\langle b_{1}, b_{2}\right\rangle \in F \times L$.

As to the second claim, just note that by Proposition 3.13 we have $\theta=I d_{L \times L}$ if and only if $\pi(\theta)=I d_{L}$.

Now we can easily obtain the following characterization:

THEOREM 4.13

Let $\mathbf{A}$ be a non-trivial algebra. Then a matrix $\langle\mathbf{A}, F\rangle$ is a reduced model of $\mathcal{L B}$ if and only if $\mathbf{A} \cong \mathbf{L} \odot \mathbf{L}$ for some lattice $\mathbf{L}$ such that the following conditions are satisfied:

1. $\mathbf{L}=\langle L, \sqcap, \sqcup\rangle$ is a distributive lattice with top element 1 satisfying the property that, for all $a, b \in L$ such that $a<b$, there is $c \in L$ such that $a \sqcup c \neq 1$ and $b \sqcup c=1$,

2. $F \cong\{1\} \times L$.

Proof. We identify $\mathbf{A}$ with its isomorphic image $\mathbf{L} \odot \mathbf{L}$, where $\mathbf{L}$ is a distributive lattice. By assumption $\mathbf{L} \odot \mathbf{L}$ is non-trivial, hence $F \neq \emptyset$. Moreover, $F \subseteq L \times L$ is an $\mathcal{L B}$-filter iff $F$ is a bifilter of $\mathbf{L} \odot \mathbf{L}$, so $F=\bar{F} \times L$ for some lattice filter $\bar{F}$ of $\mathbf{L}$. By Lemma 4.12, the matrix $\langle\mathbf{L} \odot \mathbf{L}, \bar{F} \times L\rangle$ is reduced if and only if the matrix $\langle\mathbf{L}, \bar{F}\rangle$ is reduced. As shown in [21], this last condition is equivalent to our first item plus $\bar{F}=\{1\}$.

Theorem 4.13 tells us that any $\mathbf{B} \in \mathbf{A} \lg ^{*} \mathcal{L B}$ must have a top element w.r.t. the knowledge ordering, i.e. $\top$, corresponding to $\langle 1,1\rangle \in L \times L$, where 1 is the top element of the lattice $\mathbf{L}$ such that $\mathbf{B} \cong \mathbf{L} \odot \mathbf{L}$. This also implies that $\mathbf{B}$ has a minimal nonempty bifilter, namely $\mathcal{F} \mathcal{F}(\top)=\left\{a \in B: a \geq_{t} \top\right\}$, corresponding to $\{1\} \times L$. Another interesting consequence of the theorem is that, as we have anticipated, the result of Corollary 4.7 concerning the generalized models of $\mathcal{L B}$ cannot be strengthened. That is, it is not true that if a generalized matrix $\langle\mathbf{A}, \mathcal{C}\rangle$ is a reduced generalized model of $\mathcal{L B}$, then $\mathbf{A}$ is a distributive bilattice and $\mathcal{C}$ is the family of all bifilters of $\mathbf{A}$. Consider the following:

EXAMPLE 4.14

Let $\mathbf{L}$ be any lattice that satisfies property 1 of Theorem 4.13 (for instance the the four-element non-linear distributive lattice), and let us denote its top element by 1 . 
Then we know that the matrix $\langle\mathbf{L},\{1\}\rangle$ is reduced. By Lemma 4.12, this implies that the matrix $\langle\mathbf{L} \odot \mathbf{L},\{1\} \times L\rangle$ is a reduced model of $\mathcal{L B}$. Hence, any generalized matrix $\langle\mathbf{L} \odot \mathbf{L}, \mathcal{C}\rangle$ such that $\{1\} \times L \in \mathcal{C}$ will be reduced as well. So, if we take for example $\mathcal{C}=\{\{1\} \times L, L \times L\}$, then $\langle\mathbf{L} \odot \mathbf{L}, \mathcal{C}\rangle$ is a reduced generalized model of $\mathcal{L B}$, and clearly there may be bifilters of $\mathbf{L} \odot \mathbf{L}$ that are not in $\mathcal{C}$.

The class of lattices satisfying property 1 of Theorem 4.13 seems to have some interest in itself and to deserve further study. Indeed, the literature has already considered algebras satisfying a property in some sense dual to ours, i.e. lattices having a minimum element 0 and satisfying that, for all $a, b$ such that $a>b$, there is $c$ such that $a \sqcap c \neq 0$ and $b \sqcap c=0$. This property has been called disjunction property, and the corresponding lattices disjunctive lattices [36,12]. In the same spirit, we will here adopt the name dual disjunctive for the lattices that satisfy property 1 of Theorem 4.13 .

As noted in [21], all Boolean lattices are dual disjunctive lattices in our sense. In fact, this result can be sharpened:

\section{Proposition 4.15}

Let $\mathbf{L}=\langle L, \sqcap, \sqcup\rangle$ be a Boolean lattice whose minimum and maximum element are 0 and 1 , and let $F \subseteq L$ be a filter of $\mathbf{L}$. Then the sublattice of $\mathbf{L}$ with universe $F$ is a dual disjunctive lattice.

Proof. Let $a, b \in F$ be such that $a>b$ and let $a^{\prime}$ be the complement of $a$. Clearly $a^{\prime} \sqcup b \in F$, and note that $a^{\prime} \sqcup b<1$, because otherwise we would have $a \sqcap\left(a^{\prime} \sqcup b\right)=$ $a>b=a \sqcap b=\left(a \sqcap a^{\prime}\right) \sqcup(a \sqcap b)=a \sqcap\left(a^{\prime} \sqcup b\right)$. Moreover, $a \sqcup a^{\prime} \sqcup b=1$, but $b \sqcup a^{\prime} \sqcup b=a^{\prime} \sqcup b<1$, and this completes the proof.

One may wonder if the converse of Proposition 4.15 is also true, i.e. if any dual disjunctive lattice can be proved to be isomorphic to a filter of some Boolean lattice. This is not the case, a counterexample being the following:

EXAMPLE 4.16

Let $F$ be a non-principal filter (so, without bottom element) of a Boolean lattice $\mathbf{L}=$ $\langle L, \sqcap, \sqcup\rangle$ whose maximum element is 1 . Define the structure $\mathbf{F}^{*}=\langle F \cup\{0\}, \sqcap, \sqcup, 1\rangle$, with universe $F$ augmented with a new element $0 \notin L$, and whose lattice order is the one inherited from $\mathbf{L}$, except that we have $0<a$ for all $a \in F$. Clearly $\mathbf{F}^{*}$ is a bounded distributive lattice, so if it were the filter of some Boolean lattice, it would itself be a Boolean lattice. But it is not, since for all $a, b \in F$ we have $a \sqcap b \in F$, i.e. $a \sqcap b>0$. Therefore, no element in $F$ has a complement. On the other hand, it is easy to see that $\mathbf{F}^{*}$ is dual disjunctive. Clearly if $0<a<b$ the condition is satisfied because $a, b \in F$. If $a=0$, then let $c \in F$ such that $0=a<c<b$ (such an element must exist, because $F$ had no bottom element). If we denote by $b^{\prime}$ the complement of $b$ in $\mathbf{L}$, then we have $b^{\prime} \sqcup c \in F$ and $b \sqcup b^{\prime} \sqcup c=1$, but $0 \sqcup b^{\prime} \sqcup c=b^{\prime} \sqcup c<1$. So $\mathbf{F}^{*}$ is a dual disjunctive lattice.

The results just stated allow us to gain some additional information on the class $\operatorname{Alg}^{*} \mathcal{L B}$. First of all, we may check that $\operatorname{Alg}^{*} \mathcal{L B}$ is closed under direct products but not under subalgebras (so it is not a quasivariety). The first claim follows from the fact that $\operatorname{Alg}^{*} \mathcal{L B}$ is definable by a first-order universal formula. So $P(\mathcal{F O U R}) \subseteq$ $\operatorname{Alg}^{*} \mathcal{L B}$, and by cardinality reasons we may see that this inclusion is strict, because 


\section{The logic of distributive bilattices}

there are countable algebras in $\mathbf{A l g}^{*} \mathcal{L B}$ : one just needs to consider any bilattice $\mathbf{B} \cong \mathbf{L} \odot \mathbf{L}$ where $\mathbf{L}$ is a countable Boolean lattice. The second claim can be proved by considering the nine-element distributive bilattice $\mathcal{N} \mathcal{I N \mathcal { E }}$. It is easy to see that $\mathcal{N} \mathcal{I N E}$ is isomomorphic to a subalgebra of $\mathcal{F O U R} \times \mathcal{F O U R}$, but on the other hand, as we have observed, $\mathcal{N} \mathcal{I} \mathcal{N E} \cong \mathbf{3} \odot \mathbf{3}$. Since the three-element lattice $\mathbf{3}$ is not a dual disjunctive lattice, we may conclude that $\mathcal{N} \mathcal{I N \mathcal { E }} \notin \operatorname{Alg}^{*} \mathcal{L B}$. This in turn implies that $\mathbf{A l g}^{*} \mathcal{L B} \varsubsetneqq \mathbf{A} \lg \mathcal{L B}$. Let us note again that it is significant that in the case of $\mathcal{L B}$ these two classes do not coincide, as well as the fact that $\operatorname{Alg} \mathcal{L B}$, the class of distributive bilattices, is the one that seems to be associated with this logic in a more natural way. This observation seems to be somehow confirmed by the algebraizability result contained in the next subsection.

\subsection{Algebraizability of the Gentzen calculus $\mathcal{G}_{\mathcal{L B}}$}

As anticipated, since our logic is not protoalgebraic, hence not algebraizable, there is a particular interest in studying the algebraic properties of sequent calculi associated with $\mathcal{L B}$. We end our study on this issue, stating the algebraizability of the Gentzen calculus $\mathcal{G}_{\mathcal{L B}}$ introduced in Section 1 .

THEOREM 4.17

The Gentzen calculus $\mathcal{G}_{\mathcal{L B}}$ is algebraizable w.r.t. the variety DBiLat of distributive bilattices, with the following translations:

$$
\begin{aligned}
& \tau(\Gamma \triangleright \Delta)=\{\bigwedge \Gamma \wedge(\bigwedge \Gamma \otimes \bigvee \Delta) \approx \bigwedge \Gamma\}, \\
& \rho(\varphi \approx \psi)=\{\varphi \triangleright \psi, \neg \varphi \triangleright \neg \psi, \psi \triangleright \varphi, \neg \psi \triangleright \neg \varphi\} .
\end{aligned}
$$

PROOF. Using the characterization of [33, Lemma 2.5], it is not difficult (although quite long, so we leave it to the reader) to prove that the following conditions are satisfied:

1. $\Gamma \triangleright \Delta \sim \sim_{\mathcal{G}_{\mathcal{L B}}} \rho \tau(\Gamma \triangleright \Delta)$, i.e.

$$
\begin{aligned}
\Gamma \triangleright \Delta \sim \sim_{\mathcal{G}_{\mathcal{B}}}\{\bigwedge \Gamma \wedge(\bigwedge \Gamma \otimes \bigvee \Delta) \triangleright \bigwedge \Gamma, \neg(\bigwedge \Gamma \wedge(\bigwedge \Gamma \otimes \bigvee \Delta)) \triangleright \neg \bigwedge \Gamma \\
\bigwedge \Gamma \triangleright \bigwedge \Gamma \wedge(\bigwedge \Gamma \otimes \bigvee \Delta), \neg \bigwedge \Gamma \triangleright \neg(\bigwedge \Gamma \wedge(\bigwedge \Gamma \otimes \bigvee \Delta))\}
\end{aligned}
$$

2. $\varphi \approx \psi=\|_{\text {DBiLat }} \tau \rho(\varphi \approx \psi)$, i.e.

$$
\begin{aligned}
\varphi \approx \psi=\models_{\text {DBiLat }}\{\varphi \wedge(\varphi \otimes \psi) & \approx \varphi, \neg \varphi \wedge(\neg \varphi \otimes \neg \psi) \approx \neg \varphi, \\
\psi \wedge(\varphi \otimes \psi) & \approx \psi, \neg \psi \wedge(\neg \varphi \otimes \neg \psi) \approx \neg \psi\} .
\end{aligned}
$$

3. For any distributive bilattice $\mathbf{B} \in$ DBiLat, the set $R=\left\{\langle X, Y\rangle: \bigwedge X \leq_{t}\right.$ $\bigwedge X \otimes \bigvee Y\}$ is closed under the rules of our Gentzen calculus, where $X, Y \subseteq B$ are finite and non-empty.

4. For all $T \in T h \mathcal{G}_{\mathcal{L B}}$, it holds that $\theta_{T} \in \operatorname{Con}_{\text {DBiLat }}(\mathbf{F m})$, where $\theta_{T}=\{\langle\varphi, \psi\rangle \in$ $F m \times F m: \rho(\langle\varphi, \psi\rangle) \subseteq T\}$. 


\section{Acknowledgements}

We would like to express our gratitude to the members of the Barcelona Seminar on Non-Classical Logics, during which some of the results contained in this paper were first presented, for a number of useful comments. In particular, thanks to Josep Maria Font and Ramon Jansana, for their guidance and support which helped improving the paper in several ways.

\section{References}

[1] A. R. Anderson, N. D. Belnap, and J. M. Dunn. A useful four-valued logic: How a computer should think. In Entailment. The logic of relevance and necessity, volume II, pages 506-541. Princeton University Press, 1992.

[2] O. Arieli and A. Avron. Logical bilattices and inconsistent data. In Logic in Computer Science, 1994. LICS'94. Proceedings., Symposium on, pages 468-476, 1994.

[3] O. Arieli and A. Avron. Reasoning with logical bilattices. Journal of Logic, Language and Information, 5(1):25-63, 1996.

[4] O. Arieli and A. Avron. The value of the four values. Artificial Intelligence, 102(1):97-141, 1998.

[5] A. Avron. A note on the structure of bilattices. Mathematical Structures in Computer Science, 5(3):431-438, 1995.

[6] A. Avron. The structure of interlaced bilattices. Mathematical Structures in Computer Science, 6(3):287-299, 1996

[7] N. D. Belnap. How a computer should think. In G. Ryle, editor, Contemporary Aspects of Philosophy, pages 30-56. Oriel Press, Boston, 1976.

[8] N. D. Belnap. A useful four-valued logic. In J. M. Dunn and G. Epstein, editors, Modern Uses of Multiple-Valued Logic, pages 8-37. Reidel, Dordrecht-Boston, 1977.

[9] W. J. Blok and D. Pigozzi. Protoalgebraic logics. Studia Logica, 45:337-369, 1986.

[10] W. J. Blok and D. Pigozzi. Algebraizable logics, volume 396 of Mem. Amer. Math. Soc. A.M.S., Providence, January 1989.

[11] S. Burris and H. P. Sankappanavar. A course in Universal Algebra. The millennium edition, 2000.

[12] R. Cignoli. Quantifiers on distributive lattices. Discrete Mathematics, 96:183-197, 1991.

[13] J. Czelakowski. Protoalgebraic logics, volume 10 of Trends in Logic-Studia Logica Library. Kluwer Academic Publishers, Dordrecht, 2001.

[14] M. Fitting. Bilattices and the theory of truth. Journal of Philosophical Logic, 18:225-256, 1989.

[15] M. Fitting. Bilattices in logic programming. In Proceedings of the 20th International Symposium on Multiple-Valued Logic, pages 238-246, Charlotte, 1990. The IEEE Computer Society Press.

[16] M. Fitting. Bilattices and the semantics of logic programming. J. Logic Programming, 11:91-116, 1991.

[17] M. Fitting. Kleene's logic, generalized. Journal of Logic and Computation, 1:797-810, 1991.

[18] M. Fitting. Kleene's three-valued logics and their children. Fundamenta Informaticae, 20(13):113-131, 1994. Special Anniversary Issue: 20th volume of Fundamenta Informaticae.

[19] M. Fitting. Bilattices are nice things. In Self-reference, volume 178 of CSLI Lecture Notes, pages 53-77. CSLI Publ., Stanford, CA, 2006.

[20] J. M. Font. Belnap's four-valued logic and De Morgan lattices. Logic Journal of the I.G.P.L., 5(3):413-440, 1997.

[21] J. M. Font, F. Guzmán, and V. Verdú. Characterization of the reduced matrices for the $\{\wedge, \vee\}$ fragment of classical logic. Bulletin of the Section of Logic, 20:124-128, 1991.

[22] J. M. Font and R. Jansana. A general algebraic semantics for sentential logics, volume 7 of Lecture Notes in Logic. Springer-Verlag, second edition, 2009. Electronic version freely available through Project Euclid at projecteuclid.org/euclid.lnl/1235416965. 


\section{The logic of distributive bilattices}

[23] J. M. Font, R. Jansana, and D. Pigozzi. A survey on abstract algebraic logic. Studia Logica, Special Issue on Abstract Algebraic Logic, Part II, 74(1-2):13-97, 2003. With an "Update" in 91 (2009), 125-130.

[24] J. M. Font and V. Verdú. Algebraic logic for classical conjunction and disjunction. Studia Logica, Special Issue on Algebraic Logic, 50:391-419, 1991.

[25] G. A. Fraser and A. Horn. Congruence relations in direct products. Proceedings of the American Mathematical Society, 26:390-394, 1970.

[26] M. L. Ginsberg. Multivalued logics: A uniform approach to inference in artificial intelligence. Computational Intelligence, 4:265-316, 1988.

[27] M. Kondo. Filter theory of bilattices in the semantics of logic programming. Far East Journal of Mathematical Sciences, 3(2):177-190, 2001.

[28] Y. Loyer and U. Straccia. Epistemic foundation of the well-founded semantics over bilattices. Lecture notes in computer science, pages 513-524, 2004.

[29] B. Mobasher, D. Pigozzi, G. Slutzki, and G. Voutsadakis. A duality theory for bilattices. Algebra Universalis, 43(2-3):109-125, 2000.

[30] Yu M. Movsisyan. Interlaced, modular, distributive and boolean bilattices. Armenian Journal of Mathematics, 1(3):7-13, 2008.

[31] R. Nelken and N. Francez. Bilattices and the semantics of natural language questions. Linguistics and Philosophy, 25(1):37-64, 2002.

[32] A. P. Pynko. Regular bilattices. Journal of Applied Non-Classical Logics, 10(1):93-111, 2000.

[33] J. Rebagliato and V. Verdú. Algebraizable Gentzen systems and the deduction theorem for Gentzen systems. Mathematics Preprint Series 175, University of Barcelona, June 1995.

[34] Andreas Schöter. Evidential bilattice logic and lexical inference. Journal of Logic, Language and Information, 5(1):65-105, 1996.

[35] K.M. Sim. Beliefs and bilattices. Lecture Notes in Computer Science, pages 594-594, 1994.

[36] H. Wallman. Lattices and topological spaces. Ann. of Math., 39:112-126, 1938.

[37] R. Wójcicki. Matrix approach in the methodology of sentential calculi. Studia Logica, 32:7-37, 1973. 